(c) 2004 International Press

Adv. Theor. Math. Phys. 7 (2004) 951-1001

\title{
Three-dimensional Quantum
}

\section{Supergravity and Supersymmetric Spin Foam Models}

\author{
Etera R. Livine, Robert Oeckl \\ Centre de Physique Théorique, Campus de Luminy, Case 907, 13288 \\ Marseille cedex 9, France \\ livine@cpt.univ-mrs.fr, oeckl@cpt.univ-mrs.fr
}

\begin{abstract}
We show how super BF theory in any dimension can be quantized as a spin foam model, generalizing the situation for ordinary BF theory. This is a first step toward quantizing supergravity theories. We investigate in particular 3-dimensional ( $p=1, q=1$ ) supergravity which we quantize exactly. We obtain a super-Ponzano-Regge model with gauge group $\operatorname{OSp}(1 \mid 2)$. A main motivation for our approach is the implementation of fermionic degrees of freedom in spin foam models. Indeed, we propose a description of the fermionic degrees of freedom in our model. Complementing the path integral approach we also discuss aspects of a canonical quantization in the spirit of loop quantum gravity. Finally, we comment on 2+1-dimensional quantum supergravity and the inclusion of a cosmological constant.
\end{abstract}

e-print archive: http://lanl.arXiv.org/abs/hep-th/0307251 


\section{Introduction}

Spin foam models provide a non-perturbative approach to quantum gravity $[1,2,3]$. They can be motivated both, as a rigorous way of performing a covariant path integral quantization, and as emerging as histories in the (canonical) loop approach to quantum gravity. So far these approaches to quantum gravity have lived a largely separate life from the main perturbative approach, namely string theory. An obstacle to linking the two has been the fact that string theory presumes supersymmetry. In the spin foam context this would require supergroups as gauge groups, something that so far had not been realized. However, with the recent introduction of a new formalism this limitation has been overcome $[4] .^{1}$

We consider here the extension of the spin foam approach to the quantization of supergravity theories. Apart from thus building a bridge towards string theory this has important intrinsic motivations. Foremost this is the question of including matter degrees of freedom into pure gravity spin foam models. So far, various proposals have been made both for fermions $[5,6]$ and for gauge fields $[7,8]$, but no consensus yet has emerged on how to proceed. Supergravity offers a new perspective at least for fermions. On the one hand the Lagrangian automatically contains fermions, on the other hand a superfield formulation allows to "hide" these in the gauge field. The quantization we propose proceeds by taking advantage of the superfield formulation. The generalization from fields to superfields can be thought of as occurring on the level of the gauge group, which becomes a supergroup. This allows to "mimic" the quantization of ordinary gravity. The resulting models then appear as a guide as to how to encode fermions in more general situations.

Our main focus here is three-dimensional supergravity, where we show how the exact non-perturbative path integral quantization is performed. We proceed in analogy to ordinary gravity in three dimensions. There, using frame field and connection variables the Lagrangian takes the form of BF theory. This is quantized through a discretization of the path integral using methods of lattice gauge theory. The result is independent of the discretization and provides thus an exact quantization $[9,10]$.

Using superfields, three-dimensional supergravity can be written in the analogous way by employing superframe and superconnection [11]. The

\footnotetext{
${ }^{1}$ Note that it is possible in principle to handle supergroups already by adapting the formalism of Barrett and Westbury in dimension three [12] or that of Crane, Kauffmann and Yetter in dimension four [13]. This would be rather laborious however and has the crucial disadvantage that it does not generalize to higher dimensions.
} 
gauge group becomes $\operatorname{OSp}(1 \mid 2)$ in the $(p=1, q=1)$ version of supergravity. Using the formalism of circuit diagrams [4] the quantization is then analogous to that of gravity, but with the supergroup $\operatorname{OSp}(1 \mid 2)$ replacing $\mathrm{SU}(2)$.

While our quantization of supergravity is performed using the circuit diagram formalism we also exhibit the conversion into the more conventional spin foam formalism. However, we note that a naive treatment of the resulting expressions leads to potential ambiguities. This seems to be at the origin of difficulties that have previously prevented the formulation of supersymmetric models.

We also investigate the loop quantization of this supergravity theory, which is believed to provide the canonical framework corresponding to the path integral defined by the spin foam partition function. This parallels the situation for ordinary (three-dimensional) gravity and we use super-spin networks, using $\operatorname{OSp}(1 \mid 2)$ representations, instead of the usual spin networks, using $\mathrm{SU}(2)$ representations. Following the logic of loop quantum gravity, we introduce geometric operators. However, the usual length operator, constructed from the triad, is not diagonal on the super-spin network basis, and we need to introduce a super-length operator, constructed from the super-triad. Then, using the relations between $\operatorname{OSp}(1 \mid 2)$ and $\mathrm{SU}(2)$ representations, these states of quantum supergravity can be understood as superpositions of pure (quantum) gravity states.

Based on properties of the representation theory of $\operatorname{OSp}(1 \mid 2)$ we make a proposal for identifying the fermions in the spin foam. Indeed, fermions are associated to edges of the spin foam and the partition function can be understood as a sum over gravity+fermions configurations, which are defined through the identification of fermionic paths drawn on the spin foam. This framework for fermions is very similar to the loop quantum gravity point of view. Nevertheless, in the present context, the fermionic degrees of freedom are mixed together with the gravity degrees of freedom in such a way that we can see the model both as a gravity + fermions system or as sum over special superpositions -the supersymmetric ones- of pure gravity configurations.

While we concentrate in this paper on supergravity without a cosmological constant, its inclusion could be achieved similarly as with gravity (see for example $[10,14])$. That is, the cosmological constant should correspond to a $q$-deformation of the gauge group, leading to a super-version of the TuraevViro model. Indeed we present elements of such a construction at the end, using the quantum group $\operatorname{OSp}_{q}(1 \mid 2)$.

While we only consider supergravity in dimension three here, a crucial 
property of our approach is its extendibility to higher dimensions. This is again in parallel to the situation for gravity. Namely, the Lagrangian of gravity in any dimension may be written as that of BF theory plus a constraint [15]. This suggests to quantize gravity by first quantizing BF theory (which can be done exactly and non-perturbatively) and then implementing the constraints at the quantum level. This is indeed the key idea behind recent proposals for spin foam models of quantum gravity [16]. Although, the focus has, for obvious reasons, been on dimension four the same route can be taken also in higher dimensions. Importantly, there are indications for corresponding relations between supergravity theories and super BF theory (see for example [17]). This would also open the way to a non-perturbative approach to M-theory via 11 dimensional quantum supergravity. Crucially, the quantization of super BF theory presented here works in any dimension.

In Section 2, we introduce the diagrammatics necessary to define supersymmetric spin foam models. In Section 3, we explain how the quantization of BF theory leads to spin foam models and how to include graded representations in the scheme. We illustrate this with two versions of the Ponzano-Regge model: the normal one based on $\mathfrak{s u}_{2}$ standard representation theory and a graded one obtained by assigning parities to $\mathfrak{s u}_{2}$ representations (i.e. treating them as bosonic or fermionic depending on the spin). We define precisely each model and discuss the relations between the two. In Section 4, we show how three-dimensional supergravity can be written as a (supersymmetric) BF theory. We also exhibit the representation theory of $\mathfrak{o s p}(1 \mid 2)$ necessary for the quantization. Furthermore, we discuss the canonical picture and aspects of its quantization. In Section 5, we quantize three-dimensional supergravity as a Super-Ponzano-Regge model, we study its formulation in term of supersymmetric $6 \mathrm{j}$-symbols and explore its geometry trying to disentangle the fermionic degrees of freedom from the pure gravity degrees of freedom. Finally we comment on the modifications necessary for $2+1$-dimensional supergravity and for including a cosmological constant.

\section{Circuit diagrams}

In order to efficiently represent and manipulate the state sum models considered in this paper we use the formalism of circuit diagrams [4]. This is a diagrammatic language to represent natural intertwiners of groups, supergroups or quantum groups. While somewhat similar to spin network diagrams the circuit diagrams have crucial advantages in supergroup or quantum group settings. 
The proper definitions are somewhat technical as they use the language of category theory. However, we are here interested only in diagrams arising in the context of representations of groups and supergroups. ${ }^{2}$ The present section gives an introduction to circuit diagrams in this context. Furthermore, we explain their relation to spin network diagrams.

\subsection{Diagrams for graded representations}

A circuit diagram consists of lines, called wires and rectangular boxes, called cables. Each wire carries an orientation, i.e., is equipped with an arrow. It also carries the label of a representation. A wire can pass through cables, entering at the top and leaving at the bottom. Wires may have free ends, lined up on the top and bottom line of the diagram, but nowhere else.

An (open) circuit diagram represents an intertwiner (i.e., an invariant map) between representations. Say the representations labeling the wires ending at the top are $V_{1}, \ldots, V_{n}$ and the representations labeling the wires at the bottom are $W_{1}, \ldots, W_{m}$. Then the diagram represents a map $V_{1} \otimes$ $\cdots \otimes V_{n} \rightarrow W_{1} \otimes \cdots \otimes W_{m}$. For wires with an arrow pointing upward the respective representation is replaced with its dual.

In order to evaluate a circuit diagram it is necessary to decompose it into elementary diagrams. These are then pieced together horizontally and vertically to yield the complete diagram. Horizontal composition corresponds to taking the tensor product while vertical composition corresponds to composition of maps.

The elementary diagrams consisting of wire only are listed in Figure 1. Note that the lack of a wire ending at the top or bottom of a diagram means that one takes the trivial representation $\mathbf{1}$ which is identified with the complex numbers $\mathbb{C}$. Elements of $V$ and $V^{*}$ are denoted $v$ and $\phi$ respectively. The pairing $V^{*} \otimes V \rightarrow \mathbb{C}$ between a representation $V$ and its dual $V^{*}$ is denoted by $\phi \otimes v \mapsto\langle\phi, v\rangle . \quad\left\{v_{i}\right\}$ denotes a basis of $V$ and $\left\{\phi_{i}\right\}$ a dual basis of $V^{*}$. For the crossing diagram, no arrows are drawn as the specified intertwiner takes the same form for all arrow configurations.

The only other elementary diagram is the cable, see Figure 2. The intertwiner

$$
T: V_{1} \otimes \cdots \otimes V_{n} \rightarrow V_{1} \otimes \cdots \otimes V_{n}
$$

it corresponds to is the projection of the tensor product representation onto

\footnotetext{
${ }^{2}$ In terms of [4] these are circuit diagrams for symmetric categories. More precisely, they are circuit diagrams for a category of $\mathbb{Z}_{2}$ graded objects.
} 

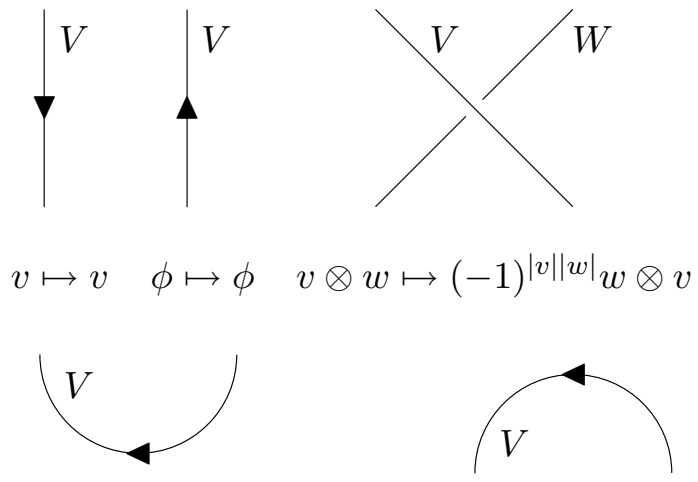

$\phi \otimes v \mapsto\langle\phi, v\rangle$

$1 \mapsto \sum_{i} v_{i} \otimes \phi_{i}$
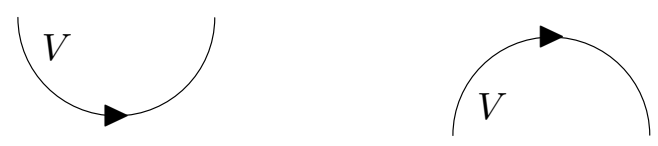

$$
v \otimes \phi \mapsto(-1)^{|v||\phi|}\langle\phi, v\rangle \quad 1 \mapsto \sum_{i}(-1)^{\left|v_{i}\right|\left|\phi_{i}\right|} \phi_{i} \otimes v_{i}
$$

Figure 1: Elementary diagrams and their corresponding intertwiners.

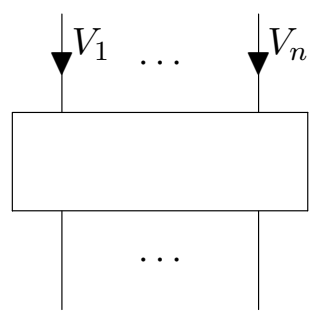

Figure 2: A cable diagram with $n$ wires going through, labelled $V_{1}$ to $V_{n}$. 
its trivial (i.e., invariant) subrepresentation. For representations of a group $G$ we can express this using the Haar measure as

$$
T: v_{1} \otimes \cdots \otimes v_{n} \mapsto \int \mathrm{d} g g \triangleright v_{1} \otimes \cdots \otimes g \triangleright v_{n} .
$$

Here $g \triangleright v$ denotes the action of $g$ on $v$.

Intuitively speaking, this is also what is going on for supergroups. To give a rigorous definition in this case, however, requires a Hopf algebraic setting. We give a rough sketch of this here without going into further details. For a group $G$ one can consider the Hopf algebra $H$ of algebraic functions on $G$. The integral then becomes a map $\int: H \rightarrow \mathbb{C}$. An action of the group becomes a coaction of $H$. This is a map $V \rightarrow V \otimes H$ written as $v \mapsto v_{(\underline{1})} \otimes v_{(2)}$. (The subscript denote indices that are summed over.) Then the intertwiner $T$ can be expressed as

$$
T: v_{1} \otimes \cdots \otimes v_{n} \mapsto v_{1(\underline{1})} \otimes \cdots \otimes v_{n(\underline{1})} \int v_{1(2)} \cdots v_{n(2)} .
$$

This is equivalent to the expression (2). However, it generalizes to the supergroup case. Namely, while a group gives rise to a commutative Hopf algebra, a supergroup gives rise to a graded commutative Hopf superalgebra $H$. The integral is again a map $\int: H \rightarrow \mathbb{C}$ and (3) again defines the intertwiner $T$ only with the difference that a factor

$$
(-1)^{\sum_{k=1}^{n} \sum_{j=2}^{n}\left|v_{j(1)}\right|\left|v_{k(2)}\right|}
$$

has to be inserted to take care of the grading.

An important property of the circuit diagrams (of the type considered here) is their invariance under "combinatorial isotopy". 3 That is, two circuit diagrams evaluate to the same intertwiner as long as they are combinatorially identical. This means they need to be composed out of the same cables and pieces of wire, connected in the same way, with the same labels, arrow directions and wire endings. However, the spatial arrangement of these components may be completely arbitrary otherwise (except for the wire endings at the top and bottom of the diagram).

A closed circuit diagram represents an intertwiner $\mathbf{1} \rightarrow \mathbf{1}$ from the trivial representation to itself, i.e., it is just a complex number. The simplest such diagram is obviously a single closed loop. Its value is easily computed

\footnotetext{
${ }^{3}$ Circuit diagrams for non-symmetric categories have weaker isotopy properties. This has the effect of placing constraints on the possible space-time dimension of spin foam models with quantum gauge groups [4].
} 
from Figure 1 by composing two opposing arches. Regardless of the arrow direction the result for a representation $V$ is ${ }^{4}$

$$
\sum_{i}(-1)^{\left|v_{i}\right|\left|\phi_{i}\right|}=\sum_{i}(-1)^{\left|v_{i}\right|}=\operatorname{dim} V_{0}-\operatorname{dim} V_{1}=\operatorname{Sdim} V
$$

i.e., the superdimension. Here, $V_{0}$ is the even and $V_{1}$ the odd part of $V=$ $V_{0} \oplus V_{1}$.

\subsection{The group case}

If we are dealing with a group the circuit diagram formalism has a simple interpretation in terms of integrals over matrix elements. Indeed the formula (2) for the cable when contracted with a dual basis of the representations $V_{1}, \ldots, V_{n}$ is just an integral over a product of matrix elements. If the circuit diagram is closed, all matrix elements are contracted to characters, i.e., traces of matrix elements.

Concretely, this implies the following rules for evaluating closed circuit diagrams of non-graded group representations [18]. Attach a group element and a direction to each cable. Associate a character with each closed loop of wire corresponding to the representation of the wire. This character is evaluated on the product of group elements associated with the cables it traverses. The product of group elements is built from right to left following the arrow direction on the wire. (The starting point is irrelevant because of the trace property.) If the directions on cable and wire are opposite the corresponding group element is replaced by its inverse. The value of the circuit diagram is the value of the complete expression with all group elements integrated over.

For graded representations of a group, similar evaluation rules hold for closed circuit diagrams. First note that formula (2) for the cable still holds. However, factors of $(-1)$ (see Figure 1) coming from the grading have to be taken into account in assembling a circuit diagram. As shown in [19] these various factors can be subsumed into a simple extra rule familiar from Feynman diagrams with fermions. Namely, the character of a fermionic representation (i.e., a fermionic loop) is to be weighted with a factor of $(-1)$. Another way to look at this is as a replacement of the character as a trace of matrix elements by the supertrace.

In the general supergroup case, such simplified rules for the evaluation of circuit diagrams no longer hold. One way to view this is as being due

\footnotetext{
${ }^{4}$ Note that $\left|v_{i}\right|=\left|\phi_{i}\right|$ for a graded basis and its dual.
} 


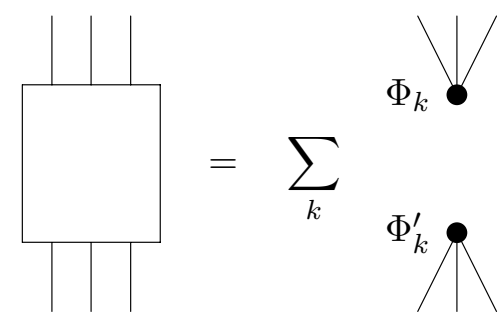

Figure 3: Decomposition of a cable into spin network nodes.

to the noncommutativity of the Hopf superalgebra of functions on the supergroup. This noncommutativity introduces ordering ambiguities for the matrix elements in the integrals.

Besides the supergroup case, the formalism also allows the extension to quantum groups. Then, more restrictive rules apply for the handling of the diagrams and a full categorical interpretation is inevitable [4]. We will not make use of this context here, but it would be the relevant one for models with a cosmological constant, see Section 5.6.

\subsection{Relation to spin networks}

We now turn to the relation between circuit diagrams and spin network diagrams. Essentially, a circuit diagram can be translated into a spin network diagram, but not necessarily the other way round. The expression of a circuit diagram in terms of spin networks is effected by a decomposition of each cable into a pair of spin network nodes, see Figure 3.

Recall that the cable represents an intertwiner (1) that projects onto the trivial subrepresentation. Decomposing this subrepresentation into onedimensional subspaces indexed by $k$ we can write

$$
T=\sum_{k} \Phi_{k}^{\prime} \Phi_{k}
$$

in terms of new intertwiners $\Phi_{k}: V_{1} \otimes \cdots \otimes V_{n} \rightarrow \mathbf{1}$ and $\Phi_{k}^{\prime}: \mathbf{1} \rightarrow V_{1} \otimes \cdots \otimes V_{n}$. These new intertwiners are now used to label the nodes arising in cutting the cable (Figure 3).

When working with spin networks one normally makes once-and-for-all choices of intertwiners to be associated with nodes for given tensor products of representations. In particular, the intertwiners are defined for arbitrary 
orientations of the legs of a node and symmetric under reordering of the legs. (Indeed this is suggested by the dot notation and familiar from the use of Feynman diagrams.) Unfortunately, this has the effect that the nice isotopy properties of the circuit diagrams are broken, in general. In other words, the value of a spin network diagram is not necessarily the same for all combinatorial ways of assembling its elements (links and nodes). This can mean that it is not well defined at all.

For non-graded group representations the problem can be and usually is fixed. A clever choice of intertwiners ensures combinatorial invariance of the spin network diagrams. For graded representations of ordinary groups the situation is already more tricky (with antisymmetries entering). For supergroups the combinatorial invariance cannot be restored. Indeed this seems to be at the root of the difficulties encountered in previous efforts to define spin networks with supergroups and to define supersymmetric spin foam models.

\section{3-Dimensional quantum gravity: two state sums}

In this section we review the path integral quantization of three-dimensional gravity. First, we review classical gravity in three dimensions and its formulation as as a BF theory. The path integral for the quantum theory, defining the dynamics of three-dimensional loop quantum gravity, is defined as a spin foam model. In this context, we start by considering the more general setting of BF-theory in arbitrary dimensions. Then specializing to gravity, we emphasize that there are two versions of the Ponzano-Regge state sum. One comes directly out of the quantization while the other results from considering half-integer representations of $\mathrm{SU}(2)$ as fermionic. This foreshadows aspects of the transition to supersymmetric models. Finally, we recall aspects of the canonical quantization.

\subsection{Classical gravity}

Let us start by describing the action for classical three-dimensional gravity in a connection formalism. Let $\mathcal{M}$ be a three dimensional manifold, the space-time. The Lorentzian (resp. Euclidean) theory is formulated in terms of two fields on $\mathcal{M}$ : a 1 -form $\omega$-the connection- valued in the Lie algebra $\mathfrak{s o}(2,1)($ resp. $\mathfrak{s o}(3))$ and a triad field $e$ valued also in $\mathfrak{s o}(2,1)$ (resp. $\mathfrak{s o}(3))$. 
Then the action is:

$$
S[\omega, e]=\int_{\mathcal{M}} \operatorname{Tr}(e \wedge F(\omega)+\Lambda e \wedge e \wedge e)
$$

where $F(w)$ is the curvature 2-form of $\omega$ and $\Lambda$ is the cosmological constant. The equations of motion impose that the curvature scalar of the connection is equal to $\Lambda$ (the connection is flat when $\Lambda=0$ ) and that the triad is compatible with the connection. The latter condition, $d_{\omega} e=0$, is the Gauss law imposing invariance under $\mathrm{SU}(1,1)$ (resp. $\mathrm{SU}(2)$ ) gauge transformations. Therefore, the theory is invariant under $\mathrm{SO}(2,1)$ (resp. $\mathrm{SO}(3)$ ) gauge transformation and under diffeomorphisms.

It is then possible to formulate it as a gauge theory of the Poincaré group [20]. For this purpose, let us introduce the 1-form

$$
A_{\mu}=\omega_{\mu}^{i} J_{i}+e_{\mu}^{i} P_{i}
$$

where $J_{i}$ are the generators of $\mathfrak{s o}(2,1)$ (resp. $\left.\mathfrak{s o}(3)\right)$ and $P_{i}$ the translation generators satisfying the following commutation relations:

$$
\begin{aligned}
& {\left[J_{i}, J_{j}\right]=\epsilon_{i j}^{k} J_{k},} \\
& {\left[J_{i}, P_{j}\right]=\epsilon_{i j}^{k} P_{k},} \\
& {\left[P_{i}, P_{j}\right]=\Lambda \epsilon_{i j}^{k} J_{k} .}
\end{aligned}
$$

$A$ is therefore valued in $\mathfrak{i s o}(2,1)$ (resp. iso $(3)$ ) when $\Lambda=0, \mathfrak{s o}(3,1)$ (resp. $\mathfrak{s o}(4))$ when $\Lambda>0$ (de Sitter case) and $\mathfrak{s o}(2,2)$ (resp. $\mathfrak{s o}(3,1))$ when $\Lambda<0$ (Anti-de Sitter case):

\begin{tabular}{|c|c|c|}
\hline & Euclidean & Lorentzian \\
\hline$\Lambda=0$ & $\mathfrak{i s o}(3)$ & $\mathfrak{i s o}(2,1)$ \\
\hline$\Lambda>0$ & $\mathfrak{s o}(4)$ & $\mathfrak{s o}(3,1)$ \\
\hline$\Lambda<0$ & $\mathfrak{s o}(3,1)$ & $\mathfrak{s o}(2,2)$ \\
\hline
\end{tabular}

Then the gravity action can be written as a Chern-Simons theory of $A$ :

$$
S[A]=\int\langle A \wedge d A\rangle+\frac{2}{3}\langle A \wedge A \wedge A\rangle
$$

where $\langle$,$\rangle is an invariant quadratic form on the \left(J_{i}, P_{i}\right)$ Lie algebra:

$$
\left\langle J_{i}, J_{j}\right\rangle=\left\langle P_{i}, P_{j}\right\rangle=0 \quad\left\langle J_{i}, P_{j}\right\rangle=\eta_{i j} .
$$

In the Euclidean $\Lambda>0$ case, using $\mathfrak{s o}(4) \sim \mathfrak{s p i n}(4) \sim \mathfrak{s u}(2) \oplus \mathfrak{s u}(2)$, we can split the connection $A$ into selfdual and antiselfdual parts $A_{ \pm}$, which are 1 -form valued in $\mathfrak{s u}(2)$. Indeed, let us introduce the generators

$$
J_{i}^{ \pm}=\frac{1}{2}\left(J_{i} \pm \frac{1}{\sqrt{\Lambda}} P_{i}\right)
$$


which satisfy the commutation relations $\left[J^{ \pm}, J^{ \pm}\right]=\epsilon J^{ \pm}$and $\left[J^{+}, J^{-}\right]=0$. Then the 1-form $A$ reads as

$$
A=\omega^{i} J_{i}+e^{i} P_{i}=A^{i+} J_{i}^{+}+A^{i-} J_{i}^{-} \quad \text { with } \quad A^{i \pm}=\omega^{i} \pm \sqrt{\Lambda} e^{i} .
$$

For $A^{ \pm}$, we can introduce a Chern-Simons action

$$
S_{ \pm}\left[A_{ \pm}\right]=\int \operatorname{Tr}_{s u(2)}\left(A_{ \pm} \wedge d A_{ \pm}+\frac{2}{3} A_{ \pm} \wedge A_{ \pm} \wedge A_{ \pm}\right)
$$

which we can combine to get the initial action

$$
S[A]=\frac{1}{4 \sqrt{\Lambda}}\left(S_{+}-S_{-}\right) .
$$

We can also do the same for the Lorentzian $\Lambda<0$ case where $\mathfrak{s o}(2,2)$ splits into $\mathfrak{s p}(2) \oplus \mathfrak{s p}(2)$ as a Lie algebra.

Finally, one can quantize the theory as a Chern-Simons theory or as the sum of two Chern-Simons theories. One can also quantize as a topological $\mathrm{BF}$ action working directly on its path integral, which leads to the PonzanoRegge-Turaev-Viro model, the spin foam model for 3-dimensional quantum gravity, which we review in the following section 3.2. The link between the Turaev-Viro model and the Chern-Simons quantization allows to track the role of the cosmological constant and relate it with the quantum deformation of the $\mathrm{SU}(2)$ group used in the spin foam model. One can also canonically quantize the theory in the spirit of loop quantum gravity [21, 22], as explained in section 3.4. It is understood to provide the canonical framework for the path integral defined by the Ponzano-Regge model, though its link with the Chern-Simons quantization is not yet clear.

\subsection{Quantization of BF theory}

We briefly recall in this section the quantization of BF theory via path integral and discretization (see for example [1]). We do this in terms of circuit diagrams on a cellular decomposition of the underlying manifold $[4,23,18]$.

Consider the BF action

$$
S[A, B]=\int_{M} \operatorname{tr}(B \wedge F),
$$

on a compact manifold $M$ of dimension $n$. Here $F$ is the curvature 2-form of a connection $A$ for a compact simple Lie group $G$. $B$ is an $n-2$-form with values in the Lie algebra of $G$. $\operatorname{tr}$ is the trace in the fundamental representation. 
As a first step to evaluate the partition function we can formally integrate out the $B$-field in the path integral as it appears linearly in the exponential

$$
\mathcal{Z}_{\mathrm{BF}}=\int \mathcal{D} A \mathcal{D} B e^{\mathrm{i} S[A, B]}=\int \mathcal{D} A \delta(F)
$$

We remain with an integral over flat connections.

To make sense of the remaining path integral we discretize the manifold $M$ as a CW-complex. In other words, we divide it into open balls ("cells") of dimension $n$. The spaces left out we fill with open balls of dimension $n-1$, the spaces again left with open balls of dimension $n-2$ etc. ${ }^{5}$ This is called a cellular decomposition of $M$.

Now what we need is the 2-skeleton of the dual complex. This means the following: Put into each $n$-cell a point, called vertex. Through each $n$-1-cell put a line, called an edge, that connects the two vertices in the adjacent $n$-cells. Finally, for each $n-2$-cell insert a surface, called a face, that is bounded by the edges which pierce the adjacent $n-1$-cells.

Now we discretize the connection $A$ as in lattice gauge theory. That is, we associate a group valued parallel transport $g_{e}$ with each edge $e$. We then measure the curvature $F$ trough the holonomies, i.e., products of parallel transports around the faces. The zero curvature condition becomes the condition that the holonomies vanish, i.e., the product of group elements around each face has to be the unit element. Finally, the integral over connections becomes the Haar measure over group elements for each edge.

The discretized version of BF theory thus has the following partition function

$$
\mathcal{Z}_{\mathrm{BF}}=\int \prod_{e} \mathrm{~d} g_{e} \prod_{f} \delta\left(g_{1} \cdots g_{k}\right)
$$

Here $g_{1}, \ldots, g_{k}$ are the group elements associated with the edges bounding the face $f$. Expanding the delta function in terms of characters of irreducible representations

$$
\delta(g)=\sum_{V} \operatorname{dim} V \chi_{V}(g)
$$

and reordering products and sums we obtain

$$
\mathcal{Z}_{\mathrm{BF}}=\sum_{V_{f}}\left(\prod_{f} \operatorname{dim} V_{f}\right) \int \prod_{e} \mathrm{~d} g_{e} \prod_{f} \chi_{V_{f}}\left(g_{1} \cdots g_{k}\right)
$$

\footnotetext{
${ }^{5}$ Think of a foam of soap bubbles: The interiors of the bubbles are the $n$-cells, the walls where two bubbles meet are the $n-1$-cells, the lines where several such walls meet are the $n-2$-cells etc.
} 


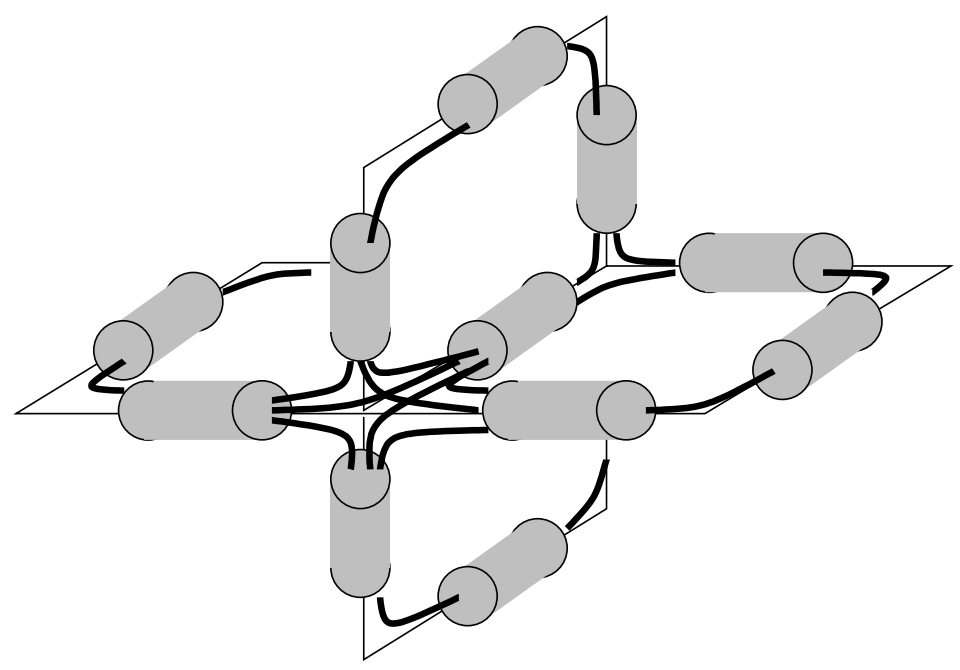

Figure 4: A piece of the circuit diagram representing the partition function of BF theory. Labels and arrows for wires are omitted.

We represent the partition function diagrammatically according to the rules of Section 2.2. Consider one big circuit diagram embedded into the manifold and constructed as follows. A closed wire is put into each face along its bounding edges. It is labelled by the representation $V_{f}$ associated with the face. A cable is put onto each edge and the wires passing along this edge are routed through the cable. This is depicted in Figure 4. We denote the value of this labeled circuit diagram by $\mathcal{D}\left(V_{f}\right)$. The value of $\mathcal{Z}_{\mathrm{BF}}$ is the value of $\mathcal{D}\left(V_{f}\right)$ summed over all assignments of irreducible representations to faces and weighted by the product of the dimensions of the representations.

For $\mathcal{Z}_{\mathrm{BF}}$ to be a good quantization of BF theory it should be independent of the chosen cellular decomposition. This is almost the case, except for an anomaly. We define the quantity ${ }^{6}$

$$
\kappa:=\sum_{V}(\operatorname{dim} V)^{2},
$$

where the sum runs over all irreducible representations $V$. The partition function is modified to read

$$
\tilde{\mathcal{Z}}_{\mathrm{BF}}:=\kappa^{p} \mathcal{Z}_{\mathrm{BF}}
$$

\footnotetext{
${ }^{6}$ Note that this quantity is infinite, except for finite groups or certain quantum groups. Nevertheless, it makes sense in the diagrammatic calculus. Also, it presumably drops out of any physically measurable quantity.
} 
where $p$ is a function of the number of cells of different dimensions. The choice of $p$ can be viewed as a renormalization problem [18] and there are different ways to fix it. For definiteness we shall $\operatorname{set}^{7}$

$$
p:=-c_{n}+c_{n-1}-c_{n-2},
$$

where $c_{k}$ is the number of $k$-cells. Let us point out that this is precisely the Euler characteristic of the 2-complex.

The discretization independence of $\tilde{\mathcal{Z}}_{\mathrm{BF}}$ was proven in dimensions 3 and 4 for simplicial decompositions in [24] and [25]. In dimension 3 it was extended to cellular decompositions in [23]. In arbitrary dimensions the proof was carried out in [18].

\subsection{Two versions of the Ponzano-Regge model}

We now turn back to the study of general relativity in three dimensions. When $\Lambda=0$, the gravity action is simply the BF-action (15) with $G=\mathrm{SO}(3)$ and $A=\omega, B=e$. Thus, the path integral quantization is given just by the partition function $\tilde{\mathcal{Z}}_{\mathrm{BF}}$ as described above. That is, it can be expressed as a sum over circuit diagrams embedded into a cellular decomposition of $M$. We shall denote the partition function in this context by $\mathcal{Z}_{\mathrm{PR}}$ so that,

$$
\mathcal{Z}_{\mathrm{PR}}=\kappa^{p} \sum_{V_{f}}\left(\prod_{f} \operatorname{dim} V_{f}\right) \mathcal{D}\left(V_{f}\right)
$$

As described in Section 2.3 we may decompose the cables into pairs of spin network nodes (Figure 3) by summing over appropriate intertwiners $\Phi_{e}$ associated with edges $e$. The state sum then acquires the form

$$
\mathcal{Z}_{\mathrm{PR}}=\kappa^{p} \sum_{V_{f}}\left(\prod_{f} \operatorname{dim} V_{f}\right) \sum_{\Phi_{e}} \prod_{v} A_{v}\left(V_{f}, \Phi_{e}\right)
$$

which is more familiar for spin foam models. Here, $A_{v}$ are the vertex amplitudes that arise as the spin networks (recoupling symbols) for the vertices. Usually, a simplicial decomposition of $M$ is employed so that the amplitudes $A_{v}$ all take the form of a $6 j$-symbol. Furthermore, as we shall assume in the following, we can use the group $G=\mathrm{SU}(2)$ instead of $\mathrm{SO}(3)$. This just means that we require $\omega$ to be a spin connection, so as to allow for the possibility of fermionic degrees of freedom. The corresponding state sum is known as the Ponzano-Regge model [9].

\footnotetext{
${ }^{7}$ Other choices (consistent with a TQFT interpretation) can be obtained by adding multiples of the Euler characteristic. A popular choice in dimension three is for example $p=-c_{0}$ [24].
} 
To be more explicit, a simplicial decomposition is a decomposition into tetrahedra. As each tetrahedron has six edges this means in terms of the dual complex that each vertex $v$ is part of six faces $f$. Thus there are six representation labels $j_{1}, \ldots, j_{6}$ associated with each vertex. Furthermore, 2cells are triangles and thus have three 1-cells in their boundary. Dually this means that any edge bounds three faces. As a consequence, there are exactly three wires going through each cable. For the group $\mathrm{SU}(2)$ (or $\mathrm{SO}(3)$ ) a tensor product of three irreducible representations has an invariant subspace of dimension at most one. Thus, the sums (5) arising in the decomposition of the cables (Figure 3) have only one summand (or none) and we can drop the intertwiner labels $\Phi_{e}$ in (21). $A_{v}$ only depends on $j_{1}, \ldots, j_{6}$ and as a spin network it takes the form of a tetrahedron. Its value is the $6 j$-symbol and the partition function takes the well known explicit form

$$
\mathcal{Z}_{\mathrm{PR}}=\kappa^{p} \sum_{j_{f}}\left(\prod_{f}\left(2 j_{f}+1\right)\right) \prod_{v}\left\{\begin{array}{lll}
j_{1} & j_{2} & j_{3} \\
j_{4} & j_{5} & j_{6}
\end{array}\right\} .
$$

A crucial point is that the representation of the partition function $\mathcal{Z}_{\mathrm{PR}}$ both in terms of circuit diagrams and in terms of recoupling symbols does not directly refer to the gauge group $G$. Instead, only the representations of $G$ appear. This allows us to modify the representation structure in a way that is not induced by choosing a different group $G$. For the group $\mathrm{SU}(2)$ there is the standard choice considered so far. But there is also the choice of introducing a grading on representations so that half-integer representations are odd. Indeed, this is what the spin-statistics relation of quantum field theory requires, if the group $\mathrm{SU}(2)$ is to play the role of the covering group of the rotations in 3-space. As this is the case in the the Ponzano-Regge model we shall adopt this grading. Mathematically one might view this as choosing a "different" version $\mathrm{SU}^{\prime}(2)$ of $\mathrm{SU}(2)$ [19]. (This also fits nicely with a categorical formulation of quantum field theory [26].)

The implementation is completely straightforward in the circuit diagram representation of the partition function. The change is captured completely by the alteration of the rules for elementary diagrams according to Figure 1. The cumulative effect of these modification is straightforward to evaluate following the rules of Section 2.2 for graded representations of groups. Namely, each closed wire labelled with an odd representation acquires a factor of -1 . Now, each representation label $V_{f}$ appears exactly twice in the circuit diagram representation, both originating from the expansion of the delta function (17). The first occurrence is in the factor $\operatorname{dim} V_{f}$, while the second is in the character $\chi_{V_{f}}$ represented as the wire going round the face $f$. The former, however, is really also a diagram, namely a closed loop of wire. It only happens to equal the dimension of the representation in the context of 
the derivation. In the more general graded case it is the superdimension (4). For the spin $j$ representation of $\mathrm{SU}(2)$ this is $(-1)^{2 j}(2 j+1)$. Thus, we have two factors of -1 for each odd representation that cancel. This proves that the graded Ponzano-Regge model and the usual one agree in their partition function. The agreement between the two models ends, however, if we insert observables that probe the signs or if we include boundaries.

To emphasize the modification we write the graded Ponzano-Regge model as

$$
\hat{\mathcal{Z}}_{\mathrm{PR}}=\hat{\kappa}^{p} \sum_{V_{f}}\left(\prod_{f} \operatorname{Sdim} V_{f}\right) \hat{\mathcal{D}}\left(V_{f}\right)
$$

This also exhibits another subtlety. Namely, in the definition (19) of $\kappa$ the dimension has to be replaced by the superdimension (hence the notation $\hat{\kappa})$. This is again because the proper definition of $\kappa$ is really as a sum over squares of loop diagrams. For the present model this doesn't make any difference as ordinary dimension and superdimension differ at most by a sign for irreducible representations.

The spin foam representation for the graded Ponzano-Regge model is obtained as usual from the circuit diagram representation. Now, the modified rules for elementary diagrams (Figure 1) have to be used. This leads (as already described) to a replacement of the dimension factors in (21) by the superdimension factors and to a modification of the $6 j$-symbols $A_{v}$. In this formulation the equality of the partition function between the two models is less easy to recognize. Indeed it seems that the two versions of the PonzanoRegge model have been occasionally confused in the literature.

We shall regard the graded model $\hat{\mathcal{Z}}_{\mathrm{PR}}$ as the physical Ponzano-Regge model. Indeed this will turn out to be relevant in the supersymmetric setting.

Writing down a partition function is not sufficient to define a quantum theory. Indeed, in the present case of three-dimensional gravity the quantum theory is really defined by extending the Ponzano-Regge model to a topological quantum field theory. This means considering manifolds with boundary. State spaces are associated to boundaries and the Ponzano-Regge state sum becomes a transition amplitude between these state spaces, see e.g. [10]. We describe this in detail in Section 5.3 in the more general supersymmetric case. 


\subsection{Canonical framework: Loop Quantum Gravity}

Complementary to the path integral approach, it is also interesting to study the canonical quantization of the BF theory formulation. This leads to threedimensional loop quantum gravity, on which the reader can can find details in the Euclidean case in [21] and in the Lorentzian context in [22].

Forgetting the cosmological constant, canonically analyzing action (6), the pairs of conjugate canonical variables are made of (the space components of) the triad $e$ and the connection $\omega$. The Hamiltonian is entirely made of constraints. The first one, $d_{\omega} e=0$, is the Gauss law imposing gauge invariance under $\mathrm{SU}(2)$ or $\mathrm{SU}(1,1)^{8}$. The second one $F=0$ can be decomposed into some space components generating space diffeomorphisms and a time component -the Hamiltonian constraint- governing the dynamics of the theory. Then one can choose $(\mathrm{SU}(2)$ or $\mathrm{SU}(1,1))$ spin networks (or more precisely equivalence classes of spin networks under space diffeomorphisms) as partial observables ${ }^{9}$, which we use as kinematical states of the quantum theory. The last step is to study their dynamics, i.e. find the states satisfying the Hamiltonian constraint. At the end of the process, one should recover gauge invariant states satisfying the $F=0$ flatness constraint.

In the framework of loop quantization, there are ambiguities on the implementation of the dynamics and we do not know yet a "good choice" of quantum Hamiltonian constraint. It turns out that spin foams allow to adress this issue: instead of canonically quantizing the Hamiltonian constraint, we construct the path integral for the theory, which provides us with a projector onto physical states solution of the Hamiltonian constraint. Indeed spin foams appear as histories of spin networks and spin foam models might be viewed as loop gravity models taking into account the dynamics. More precisely, when one looks at the boundary (or a two-dimensional slice)

\footnotetext{
${ }^{8} \mathrm{SU}(1,1) \sim \mathrm{SL}(2, \mathbb{R}) \sim \mathrm{Sp}(2)$ is the double cover of $S O(2,1)$ but it is not its universal cover. The first order formulation of gravity uses the triad and therefore needs spinor indices provided by $S U(2)$ in the Euclidean case and $S U(1,1)$ in the Lorentzian case. Moreover, representations of $\mathrm{SU}(1,1)$ are associated a parity $\epsilon= \pm 1$ (whether there are also representations of $S O(2,1)$ or not), whereas representations of the universal cover would be labelled by a continuous "parity" $\epsilon \in[0,1]$. Therefore using $S U(1,1)$ to formulate a Lorentzian quantum theory leads to structures very similar to those of the Euclidean theory.

${ }^{9}$ True Dirac observables should commute with all the constraints. Partial observables do not. Here they are quantities which are chosen to be gauge invariant (under the Gauss law) and diffeomorphism invariant. But the Hamiltonian constraint will act non-trivially on them, i.e. they have a non-trivial time evolution. That is why we call them "kinematical states". Then we would like to project this space of kinematical states onto physical states satisfying also the Hamiltonian constraints. The reader can find a discussion on the notion of partial observables and their use in [27].
} 
of a 2-complex (a spin foam) in the Ponzano-Regge model, one gets $\mathrm{SU}(2)$ spin networks (or $\mathrm{SU}(1,1)$ spin networks in its Lorentzian theory). From this point of view, the Ponzano-Regge model appears as the space-time version or path integral formulation for three-dimensional loop quantum gravity. Then one can check that the Ponzano-Regge partition function defines a projector (in the space of the boundary states, see the comments at the end of Section 3.3) onto the $F=0$ sector and thus projects onto physical states of the canonical theory $[10]$.

In the case of a cosmological constant, only the Hamiltonian constraint is modified, and the Turaev-Viro model (deformed Ponzano-Regge model) still allows to project onto states satisfying the constraints [10].

\section{3-Dimensional classical supergravity}

\subsection{Lagrangian formulation}

One can extend the formulation of three-dimensional gravity, described in the previous section, to the supersymmetric case of supergravity. This is achieved in the Lorentzian Anti-de Sitter case by extending the symmetry group $\operatorname{Sp}(2) \times \operatorname{Sp}(2)$ to $\operatorname{OSp}(p \mid 2) \times \operatorname{OSp}(q \mid 2)$. These $(p, q)$ type $\mathrm{AdS}$ supergravities were introduced by Achúcarro and Townsend [11]. The same procedure works in the Euclidean de Sitter case and we extend $\mathrm{SU}(2) \times \mathrm{SU}(2)$ to $\operatorname{OSp}_{E}(p \mid 2) \times \operatorname{OSp}_{E}(q \mid 2)$ (where the subscript $E$ means that the bosonic part of the supergroup is $O(p) \times \mathrm{SU}(2)$ and not $O(p) \times \mathrm{Sp}(2))$.

In the present paper, we are interested in the simplest supergravity case $(p=1, q=1)$ with a $\operatorname{OSp}_{E}(1 \mid 2) \times \operatorname{OSp}_{E}(1 \mid 2)$ supersymmetry. Using $\mathfrak{s u}(2)$ spinor indices $A, B, . .= \pm$ and the notation $(A B)$ (resp. $[A B]$ ) for symmetrising (resp. antisymmetrising) the couple of indices $A B$, the superalgebra $\mathfrak{o s p}_{E}(1 \mid 2)$ reads

$$
\begin{aligned}
{\left[J_{A B}, J^{C D}\right] } & =\delta_{[A}^{[C} J_{B]}^{D]}, \\
\left\{Q_{A}, Q_{B}\right\} & =J_{A B}, \\
{\left[J_{A B}, Q_{C}\right] } & =\epsilon_{C(A} Q_{B)} .
\end{aligned}
$$

Then, one can introduce the supertriad

$$
\mathcal{E}=e^{A B} J_{A B}+\phi^{A} Q_{A}
$$

and a 1 -form $\mathcal{A}$ valued in $\mathfrak{o s p}_{E}(1 \mid 2)$-the superconnection:

$$
\mathcal{A}=\omega^{A B} J_{A B}+\psi^{A} Q_{A},
$$


whose curvature is defined as $\mathcal{F}=\widetilde{F}^{A B} J_{A B}+F^{A} Q_{A}$ with components

$$
\begin{aligned}
\widetilde{F}^{A B} & =F^{A B}(\omega)+\psi^{A} \wedge \psi^{B}, \\
F^{A} & =d_{\omega} \psi^{A}=d \psi^{A}+\omega_{B}^{A} \wedge \psi^{B} .
\end{aligned}
$$

The supersymmetric BF action for supergravity then reads

$$
S_{\text {sugra }}[\mathcal{E}, \mathcal{A}]=\int_{\mathcal{M}} \operatorname{STr}(\mathcal{E} \wedge \mathcal{F}(\mathcal{A})+\Lambda \mathcal{E} \wedge \mathcal{E} \wedge \mathcal{E})
$$

The equations of motion are $\mathcal{F}+\Lambda \mathcal{E} \wedge \mathcal{E}=0$ imposing that the curvature scalar is $\Lambda$ and the super Gauss law $d_{\mathcal{A}} \mathcal{E}=0$ imposing the ordinary $\mathrm{SU}(2)$ gauge invariance and the (left-handed) supersymmetric constraint.

Introducing the selfdual and antiselfdual components makes the $\mathfrak{o s p}_{E}(1 \mid 2) \oplus$ $\mathfrak{o s p}_{E}(1 \mid 2)$ symmetry explicit. Let us define

$$
\begin{aligned}
A_{ \pm}^{A B} & =\omega^{A B} \pm \sqrt{\Lambda} e^{A B} \\
\psi_{ \pm}^{A} & =\psi^{A} \pm \Lambda^{1 / 4} \phi^{A} .
\end{aligned}
$$

Then the supergravity theory can be expressed as the sum of two ChernSimons theories on the 1 -forms valued in $\mathfrak{o s p}_{E}(1 \mid 2), A_{+}^{A B} J_{A B}+\psi_{+}^{A} Q_{A}$ and $A_{-}^{A B} J_{A B}+\psi_{-}^{A} Q_{A}$, with the two fermionic fields $\psi_{ \pm}$:

$$
S_{\text {sugra }}=\frac{1}{\sqrt{\Lambda}} \int_{\mathcal{M}} I_{C S}\left(A_{+}^{A B} J_{A B}+\psi_{+}^{A} Q_{A}\right)-I_{C S}\left(A_{-}^{A B} J_{A B}+\psi_{-}^{A} Q_{A}\right)
$$

with

$I_{C S}\left(A_{ \pm}, \psi_{ \pm}\right)=S \operatorname{Tr}_{\text {osp }_{E}(1 \mid 2)}\left(A_{ \pm} \wedge d A_{ \pm}+\frac{2}{3} A_{ \pm} \wedge A_{ \pm} \wedge A_{ \pm}+\psi_{ \pm} \wedge d_{A_{ \pm}} \psi_{ \pm}\right)$

which is the form in which the supergravity theories are introduced in [11].

Using this formalism, we are going to quantize three-dimensional supergravity as a super Ponzano-Regge model with symmetry group $\operatorname{OSp}_{E}(1 \mid 2)$ instead of $\mathrm{SU}(2)$. This state sum model will be a topological theory based on the representations of the superalgebra $\mathfrak{o s p}_{E}(1 \mid 2)$ and, in the framework of spin foam models, it should implement a (discrete) path integral for gravity plus fermions in three dimensions.

\subsection{Representation theory of $\mathfrak{o s p}_{E}(1 \mid 2)$}

Instead of using the supergroup $\operatorname{OSp}_{E}(1 \mid 2)$ directly we shall use the super Lie algebra $\mathfrak{o s p}_{E}(1 \mid 2)$ to obtain its representations. This is technically easier and allows the recurse to well established results in the literature. We review these in the present section. 


\subsubsection{Generators and action}

To study the representations of $\mathfrak{o s p}_{E}(1 \mid 2)$, it is useful to write the algebra as follows ${ }^{10}$ :

$$
\begin{array}{rrr}
{\left[J_{3}, J_{ \pm}\right]= \pm J_{ \pm}} & {\left[J_{+}, J_{-}\right]=2 J_{3}} \\
{\left[J_{3}, Q_{ \pm}\right]= \pm \frac{1}{2} Q_{ \pm} \quad\left[J_{ \pm}, Q_{ \pm}\right]=0} & {\left[J_{ \pm}, Q_{\mp}\right]=Q_{ \pm}} \\
\left\{Q_{ \pm}, Q_{ \pm}\right\}= \pm \frac{1}{2} J_{ \pm} & \left\{Q_{\mp}, Q_{ \pm}\right\}=-\frac{1}{2} J_{3}
\end{array}
$$

The Casimir operator is $C=J^{i} J^{i}+Q_{+} Q_{-}-Q_{-} Q_{+}$. The representations [29] are labelled by half-integer $j$ and are made out of pairs of $\mathfrak{s u}(2)$ representations carrying spin $j$ and $j-1 / 2$ :

$$
R^{j}=V^{j} \oplus V^{j-\frac{1}{2}} \quad \forall j \geq \frac{1}{2} .
$$

We call $j$ the spin of the $\mathfrak{o s p}(1 \mid 2)$ representations, $k=j, j-1 / 2$ the two isospins, and we label $|j, k, m\rangle$ the vectors of a basis of $R^{j}$ using the usual notations for $\mathfrak{s u}(2)$. More precisely, the action of the generators in the $j$ representations is:

$$
\begin{aligned}
J_{3}|j, j, m\rangle & =m|j, j, m\rangle, \\
J_{3}\left|j, j-\frac{1}{2}, m\right\rangle & =m\left|j, j-\frac{1}{2}, m\right\rangle, \\
J_{ \pm}|j, j, m\rangle & =((j \mp m)(j \pm m+1))^{1 / 2}|j, j, m \pm 1\rangle, \\
J_{ \pm}\left|j, j-\frac{1}{2}, m\right\rangle & =\left(\left(j-\frac{1}{2} \mp m\right)\left(j+\frac{1}{2} \pm m\right)\right)^{1 / 2}\left|j, j-\frac{1}{2}, m \pm 1\right\rangle, \\
Q_{ \pm}|j, j, m\rangle & =\mp(j \mp m)^{1 / 2}\left|j, j-\frac{1}{2}, m \pm \frac{1}{2}\right\rangle, \\
Q_{ \pm}\left|j, j-\frac{1}{2}, m\right\rangle & =-\frac{1}{2}\left(j+\frac{1}{2} \pm m\right)^{1 / 2}\left|j, j, m \pm \frac{1}{2}\right\rangle .
\end{aligned}
$$

In fact, we have two levels $V^{j}$ and $V^{j-1 / 2}$. The $\mathfrak{s u}(2)$ generators $J_{3, \pm}$ act as usual on each level. Then the supersymmetric generators allow to go from one level to the other. The Casimir of the $R^{j}$ representation is

\footnotetext{
${ }^{10}$ In terms of the $\mathfrak{s u}(2)$ generators $J_{1,2,3}$, the algebra reads

$$
\left[J_{i}, Q_{A}\right]=\left(\gamma_{i}\right)_{A}^{B} Q_{B} \quad\left\{Q_{A}, Q_{B}\right\}=\left(\gamma_{i}\right)_{A B} J_{i}=\left(\epsilon \gamma_{i}\right)_{A}^{B} J_{i}
$$

where the $\gamma_{i}$ are the Pauli matrices and $\epsilon$ the antisymmetric matrix:

$$
\left(\gamma_{1}\right)_{A}^{B}=\frac{1}{2}\left(\begin{array}{cc}
0 & 1 \\
1 & 0
\end{array}\right) \quad \gamma_{2}=\frac{i}{2}\left(\begin{array}{cc}
0 & -1 \\
1 & 0
\end{array}\right) \quad \gamma_{3}=\frac{1}{2}\left(\begin{array}{cc}
1 & 0 \\
0 & -1
\end{array}\right) \quad \epsilon=\left(\begin{array}{cc}
0 & 1 \\
-1 & 0
\end{array}\right) .
$$
}




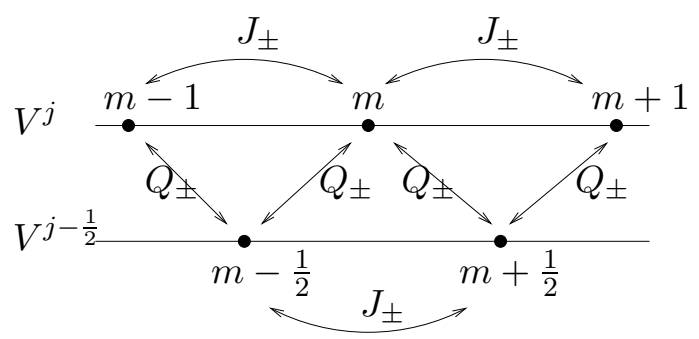

Figure 5: Structure of the $R^{j}$ representation of $\mathfrak{o s p}(1 \mid 2)$.

$$
C_{j}=j\left(j+\frac{1}{2}\right)
$$

So the fermions contribution is $-\frac{j}{2}$ on the subspace $V^{j} \hookrightarrow R^{j}$ and $\frac{1}{2}\left(j+\frac{1}{2}\right)$ on $V^{j-1 / 2}$.

\subsubsection{Parity}

Due to the "anti-commuting" properties of the $Q$ generators, we cannot define a simple star operator and talk about unitary representations. We need to introduce the parity (bosonic or fermionic) of the vectors of the representations, use a grade star operation and talk about grade-star representations [29].

In a given representation $R^{j}$, we introduce a parity $\lambda=0,1$ which means that the vectors $|j, j, m, \lambda\rangle$ of $V^{j}$ have parity $\lambda$ ( 0 for bosonic and 1 for fermionic) and that the vectors $|j, j-1 / 2, m, \lambda+1\rangle$ of $V^{j-1 / 2}$ have parity $\lambda+1$.

The grade star operation ${ }^{\dagger}$ will take into account the degree of the operators/vectors (whether it is bosonic or fermionic). More precisely, given an operator $A$ of degree $\alpha, A^{\dagger}$ is defined as:

$$
\left\langle A^{\dagger} \psi \mid \varphi\right\rangle=(-1)^{\alpha \xi}\langle\psi \mid A \varphi\rangle \quad \text { with } \xi \text { the degree of } \psi \text {. }
$$

With this definition we can require the hermiticity relations:

$$
J_{i}^{\dagger}=J_{i} \quad Q_{+}^{\dagger}=\mp Q_{-} \quad Q_{-}^{\dagger}= \pm Q_{+} .
$$

Hermiticity of the $J$ generators imposes that

$$
\begin{aligned}
\left\langle j, j, m, \lambda \mid j, j, m^{\prime}, \lambda\right\rangle & =g \delta_{m, m^{\prime}} \\
\left\langle j, j-\frac{1}{2}, m, \lambda+1 \mid j, j-\frac{1}{2}, m^{\prime}, \lambda+1\right\rangle & =h \delta_{m, m^{\prime}}
\end{aligned}
$$


where $g, h$ are just signs. Hermiticity of the $Q$ generators then implies $h=$ $\pm(-1)^{\lambda} g$. Finally, we have a grade star representation with a positive definite scalar product iff $g=h$, i.e. $\pm(-1)^{\lambda}=1$ (with \pm defined in (37)).

So far every irreducible representation $R^{j}$ appears twice, once for each choice of parity. However, we might wish to allow only one parity to occur. Indeed, if the sub Lie algebra $\mathfrak{s u}_{2} \subset \mathfrak{o s p}(1 \mid 2)$ plays the physical role of (infinitesimal) rotations the representations must obey the spin-statistics relation of quantum field theory. That is, the $V_{j}$ should be even or odd depending on whether $j$ is integer or not. In the Hopf algebraic approach to supergroups it is actually possible to encode this restriction in the supergroup [28]. There is an ordinary (not super but noncommutative) Hopf algebra that encodes $\operatorname{OSp}(1 \mid 2)$. This has representations of both parities. Then there are restricted Hopf algebras which allow for each irreducible representation to occur with only one parity. The version we consider here is called $\operatorname{OSp}^{\prime}(1 \mid 2)$ in $[28] .{ }^{11}$ We shall continue to pursue the super Lie algebraic point of view, but our remark ensures that the restriction of the parity is consistent. Technically speaking it means that we have a "good" category of representations.

The implications of the restriction in parity are as follows. For $j \in \mathbb{N}$, $Q_{+}^{\dagger}=-Q_{-}$and $Q_{-}^{\dagger}=Q_{+}$on $R^{j}$ and, for $j \in \mathbb{N}+1 / 2, Q_{+}^{\dagger}=Q_{-}$and $Q_{-}^{\dagger}=-Q_{+}$on $R^{j}$. The superdimension of the representation is given by the supertrace of the identity and yields:

$$
\operatorname{Sdim}\left(R^{j}\right)=(-1)^{2 j} \operatorname{dim}\left(V^{j}\right)-(-1)^{2 j} \operatorname{dim}\left(V^{j-1 / 2}\right)=(-1)^{2 j} .
$$

\subsubsection{Tensor products}

Let us now describe the tensor products of representations $R^{j}$. Taking into account that $R^{j}=V^{j} \oplus V^{j-1 / 2}$ and the recoupling theory of $\mathfrak{s u}(2)$ representations, we get:

$$
R^{j_{1}} \otimes R^{j_{2}}=\bigoplus_{\left|j_{1}-j_{2}\right| \leq j \leq\left(j_{1}+j_{2}\right)} R^{j},
$$

where the sum over $j$ goes by half-integer steps. Therefore, we see that the situation is very similar to the $\mathfrak{s u}(2)$ case with a triangular inequality on the

\footnotetext{
${ }^{11}$ There is another subtlety which has not been sufficiently appreciated in the literature. $\mathrm{OSp}_{E}(1 \mid 2)$ is supposed to have a bosonic subgroup $\mathrm{SU}(2) \times O(1)$ with $O(1)=\mathbb{Z}_{2}$ (and this is indeed the case of $\operatorname{OSp}(1 \mid 2)$ in [28]). However the representation theory of $\mathfrak{o s p}(1 \mid 2)$ as considered here does not allow for an appropriate $\mathbb{Z}_{2}$ action. This means that it really corresponds to some "truncated" version of $\operatorname{OSp}(1 \mid 2)$. Nevertheless we shall be content with this truncation since it is self-consistent and the bosonic subgroup $\mathrm{SU}(2)$ is properly contained.
} 
representations and the only difference is that the representation $j$ takes all half-integer values and not only integer steps as in the representation theory of $\mathfrak{s u}(2)$. As an example, we now have

$$
R^{\frac{1}{2}} \otimes R^{\frac{1}{2}}=R^{0} \oplus R^{\frac{1}{2}} \oplus R^{1}
$$

instead of $V^{1 / 2} \otimes V^{1 / 2}=V^{0} \oplus V^{1}$. Then the intertwiner $I^{j_{1} j_{2}}{ }_{j_{3}}: R^{j_{1}} \otimes R^{j_{2}} \rightarrow$ $R^{j_{3}}$ is unique (up to normalization) and we can deduce the corresponding (supersymmetric) Clebsh-Gordan coefficients expressing the change of basis between the vectors $\left|j_{1} k_{1} m_{1}\right\rangle \otimes\left|j_{2} k_{2} m_{2}\right\rangle$ and $\left|\left(j_{1}, j_{2}\right) j_{3} k_{3} m_{3}\right\rangle$.

The simplest way to get the recoupling coefficients is to work with the isospin decomposition $R^{j}=V^{j} \oplus V^{j-\frac{1}{2}}$ and use the known $\mathfrak{s u}(2)$ ClebshGordan coefficients. Then the restriction of the intertwiner of the $k_{1}=$ $j_{1}, k_{2}=j_{2}$ isospins $I^{j_{1} j_{2}} j_{3}: V^{j_{1}} \otimes V^{j_{2}} \rightarrow V^{j_{3}} \oplus V^{j_{3}-\frac{1}{2}}$ is invariant under $S U(2)$. Therefore either the image is $V^{j_{3}}$ or $V^{j_{3}-\frac{1}{2}}$ depending whether $j_{1}+$ $j_{2}+j_{3}$ is an integer or not.

This way, we see that they are two different situations:

- $j_{1}+j_{2}+j_{3} \in \mathbb{N}$ :

The only non-vanishing components are $V^{j_{1}} \otimes V^{j_{2}} \rightarrow V^{j_{3}}$, which is the usual $\mathfrak{s u}(2)$ intertwiner and its supersymmetric counterparts $V^{j_{1}-\frac{1}{2}} \otimes V^{j_{2}} \rightarrow V^{j_{3}-\frac{1}{2}}$ and $V^{j_{1}} \otimes V^{j_{2}-\frac{1}{2}} \rightarrow V^{j_{3}-\frac{1}{2}}$. Each of these intertwiners are given up to a factor by the $\mathfrak{s u}(2)$ Clebsh-Gordan coefficients. Then their relative normalization is fixed by invariance of $I^{j_{1} j_{2}} j_{3}$ under the supersymmetric generators $Q_{ \pm}$, so that $I^{j_{1} j_{2}} j_{3}$ is unique up to an overall factor. One can find the explicit expressions of the relative factors in [29].

- $j_{1}+j_{2}+j_{3} \in \mathbb{N}+\frac{1}{2}$ :

The only non-vanishing components are $V^{j_{1}-\frac{1}{2}} \otimes V^{j_{2}-\frac{1}{2}} \rightarrow V^{j_{3}-\frac{1}{2}}$, and its supersymmetric counterparts $V^{j_{1}} \otimes V^{j_{2}} \rightarrow V^{j_{3}-\frac{1}{2}}, V^{j_{1}-\frac{1}{2}} \otimes V^{j_{2}} \rightarrow$ $V^{j_{3}}$ and $V^{j_{1}} \otimes V^{j_{2}-\frac{1}{2}} \rightarrow V^{j_{3}}$. Once again, each of these intertwiners is given up to a factor by the $\mathfrak{s u}(2)$ Clebsh-Gordan coefficient and their relative normalization is fixed by the invariance under the supersymmetry generators. $I^{j_{1} j_{2}}{ }_{j 3}$ is then unique up to an overall normalization. 


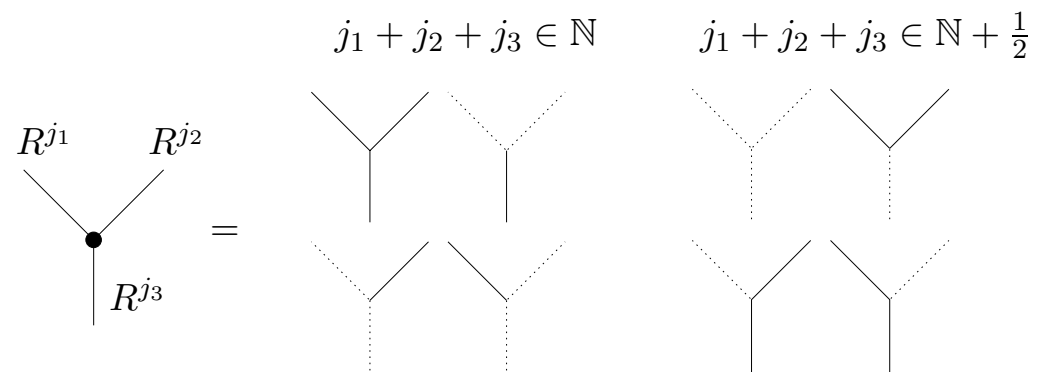

Figure 6: Intertwiner $I^{j_{1} j_{2}} j_{3}: R^{j_{1}} \otimes R^{j_{2}} \rightarrow R^{j_{3}}$ invariant under osp $(1 \mid 2)$ : the decomposition on isospins $V^{j_{i}}$ (solid lines) and $V^{j_{i}-1 / 2}$ (dotted lines) in the two cases $j_{1}+j_{2}+j_{3} \in \mathbb{N}$ and $j_{1}+j_{2}+j_{3} \in \mathbb{N}+\frac{1}{2}$.

\section{3-Dimensional quantum supergravity}

\subsection{A topological super Ponzano-Regge model}

As the starting point for the quantization we take the BF-type formulation of 3-dimensional supergravity (28), without cosmological constant. In order to perform the quantization we wish to follow the same recipe as in Section 3.2. Superficially, everything seems to go through (cellular decomposition, parallel transports etc.), if we simply replace the group $\mathrm{SU}(2)$ by the supergroup $\operatorname{OSp}(1 \mid 2)$. However, there is a problem. An expression such as (16) or (18) is no longer well defined since we encounter ordering ambiguities between functions on the supergroup as they no longer commute. On the other hand, the corresponding circuit diagram representation of the partition function is still well defined [4]. Furthermore, fixing the anomaly one obtains again a partition function that does not depend on the chosen cellular decomposition $[4,23]$.

Thus, we obtain a good quantization which we write as

$$
\mathcal{Z}_{\text {sugra }}=\hat{\kappa}^{p} \sum_{V_{f}}\left(\prod_{f} \operatorname{Sdim} V_{f}\right) \hat{\mathcal{D}}\left(V_{f}\right)
$$

As before, the hats indicate that the quantities involved carry nontrivial gradings. The sum is now a sum over irreducible representations $V_{f}$ of $\operatorname{OSp}(1 \mid 2)$ for each face $f$. More precisely, as explained in Section 4.2, we choose only those representations that obey the spin-statistics relation. This means, we effectively restrict to the reduced supergroup $\operatorname{OSp}^{\prime}(1 \mid 2)$ [28] (see Section 4.2). The bosonic theory which is extended is the graded PonzanoRegge model (23) and not the non-graded one (20). 
In the following we shall consider the appropriate representations of $\mathfrak{o s p}(1 \mid 2)$ as discussed in Section 4.2 (see footnote 11).

\subsection{Decomposition into supersymmetric $6 j$-symbols}

It would be desirable to obtain also in the supersymmetric case a formulation of the model in terms of the standard spin foam language, paralleling the usual formulation of the ordinary Ponzano-Regge model (22). Roughly speaking, we wish to decompose the cables into intertwiners (Figure 3) as in Section 3.3, to obtain a sum over products of $6 j$-symbols associated with the tetrahedra of a simplicial decomposition.

Superficially, the procedure seems to be the same as already sketched in Section 3.3. There is a crucial difference, however. In contrast to the $\mathfrak{s u}(2)$ case the $3 j$-symbols for $\mathfrak{o s p}(1 \mid 2)$ that replace the cable (Figure 3 ) no longer have a purely combinatorial definition. In other words, the meaning of a $3 j$-symbol now depends on its graphical representation. Another way to say this is that the $3 j$-symbols for $\mathfrak{o} \mathfrak{s p}(1 \mid 2)$ do not enjoy the same symmetry properties (under moving around and exchange of legs) as the ones for $\mathfrak{s u}(2)$. This dependence on the graphical representation is inherited by the $6 j$-symbols composed of the $3 j$-symbols. One might view this as causing an information loss in going to a description such as (22). This phenomenon is well known when employing quantum groups. Indeed, in the case of the Ponzano-Regge model it is not sufficient to replace $6 j$-symbols with quantum $6 j$-symbols. There is crucial topological information which is not captured by a formula of the type (22). Although it is common to write down such a formula also in the quantum group case it is then implicitly understood that this is not a complete definition in itself. Unfortunately, this fact does not seem to be universally appreciated in the physics community.

The same situation (although in a sense less severe) occurs for supergroups. Thus, although definitions of $6 j$-symbols for $\mathfrak{o s p}(1 \mid 2)$ do exist [30], it would not be sufficient to write a naive formula as (22) with those to get a well defined model. In contrast to the $\mathfrak{s u}(2)$ case additional topological information must be specified. It is a crucial advantage of the circuit diagram formalism that it does not suffer from this problem but encodes the complete information [4]. Thus, when splitting up cables we need to keep track of this information. In the following we describe this splitting in more detail.

The procedure consists of a few steps: 


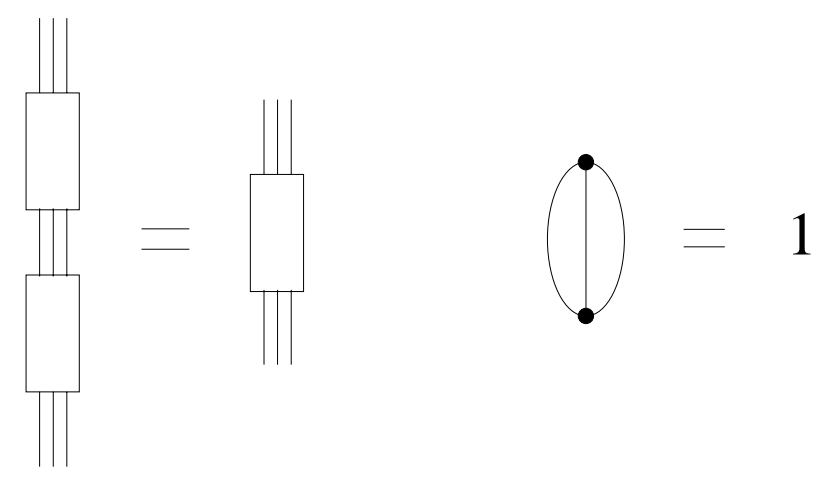

Figure 7: The projection property of the cables implies a normalization condition for $3 j$-symbols. The normalization of the $\Theta$ net to one is valid for all choices of orientations of the links.

- First, we choose arrows (orientations) for each face of the dual 2complex (i.e. the spin foam), allowing to distinguish a representation from its dual.

- Then we cut each cable in two, decomposing it into pairs of $3 j$ symbols following Figure 3. Generically, a cable would decompose into a sum of such pairs. But in the case of $\mathfrak{o s p}(1 \mid 2)$, as in the $\mathfrak{s u}(2)$ case, there is one unique intertwiner up to normalization between a tensor product of three irreducible representations. A cable decomposes simply into two $3 j$ symbols.

Each $3 j$ symbol corresponds to a map $R^{j_{1}} \otimes R^{j_{2}} \otimes R^{j_{3}} \rightarrow \mathbb{C}$, or $R^{j_{1}} \otimes R^{j_{2}} \otimes\left(R^{j_{3}}\right)^{*} \rightarrow \mathbb{C}$ etc. depending on the arrows of the edges around the cable. These invariant tensors can all be constructed up to normalization from the tensor product decomposition, defining the maps $R^{j_{1}} \otimes R^{j_{2}} \rightarrow R^{j_{3}}$.

Then, of course, there is the issue of the normalization of these $3 j$ symbols. A key property of the cables (which is crucial for the discretization independence) is that the composition of two cables is equivalent to a single cable (see Figure 7). This translates into a normalization of the $3 j$ symbols such that the contraction of the two $3 j$ symbols is equal to 1 (see Figure 7 ). Note that all the individual $3 j$ symbols are defined with respect to a fixed graphical representation.

- After having decomposed the cables associated to the edges, we gather and contract the $3 j$ symbols around each vertex (i.e. tetrahedron) and evaluate the resulting diagram thus defining a $6 j$ symbol. Crucially, in this process the rules of Section 2 apply, in particular those of Figure 1. 
Then the partition function is the product of all these $6 j$ symbols, together with the measure on the representation defined by the superdimension.

At the end of the day, we obtain a partition function for a closed manifold written as

$$
\mathcal{Z}_{\text {sugra }}=\sum_{j_{f}}\left(\prod_{f}(-1)^{2 j_{f}}\right) \prod_{v}\{6 j\}_{\text {susy }}
$$

Note that in the process we have explicitly incorporated the topological matching information into the $6 j$-symbols. In particular, the $6 j$-symbols are not necessary defined uniformly. That is, $6 j$-symbols associated with different tetrahedra might have a priori different normalizations, even if they are labeled with the same representations. Given the results of [30] it is probable that this nonuniformity can be removed up to sign factors. While that would be clearly desirable, it goes beyond the scope of this paper.

Let us point out that the weight for each representation is simply a sign $(-1)^{2 j}$ compared to the $\mathfrak{s u}(2)$ weight of modulus $(2 j+1)$. The supersymmetric partition function is thus likely to be less divergent than the classical Ponzano-Regge partition function (which needs a regularization). It would be a strong result if this new state sum was convergent since it would mean that supersymmetry regulates the gravity partition function. However, the divergences of the classical Ponzano-Regge model have been shown to come from the diffeomorphism invariance [31] and there is no reason to expect that this gauge invariance will not make the supersymmetric state sum (40) diverge. This issue would be analysed carefully, nevertheless it is likely that the proposed partition function will need to be regularized either by a cut-off (as in the original proposal by Ponzano and Regge), or by going to quantum super-groups (see section 5.6 for more details) or by gauge fixing (as proposed in [31]).

\subsection{Boundaries and TQFT}

So far we have only concerned ourselves with the partition function. For a full-fledged quantum theory we need to specify states, observables etc. We follow here the approach of topological quantum field theory (TQFT) which is well established for three-dimensional quantum gravity [24, 10]. Concretely, we consider 3-manifolds with boundaries. State spaces are associated with these boundaries while linear maps between them are associated with the 3-manifolds. The construction for quantum supergravity proceeds 
essentially identically to the construction for quantum gravity. Nevertheless, we briefly recall it here to highlight differences that arise.

Let $M$ be a compact three-dimensional manifold with boundary $\partial M$. We consider a cellular decomposition of $M$. This implies in particular a cellular decomposition of $\partial M$. Consider the dual 1-complex on $\partial M$, consisting of nodes (dual to 1-cells) and links (dual to 0-cells). This 1-complex bounds the 2-complex in the interior of $M$ that is dual to the cellular decomposition of $M$. For a given labeling of faces and edges of the 2-complex in $M$ (in the picture where cables are decomposed as in Section 5.2) we get an induced labeling of links and nodes on the boundary. In other words, we obtain a spin foam in the interior bounded by a spin network on the boundary [32]. The details of this construction in terms of the circuit diagram formalism are described in [4].

A given cellular decomposition $C$ of a 2-manifold $\Sigma$ thus yields a vector space $H_{\Sigma, C}$, namely the space with basis given by all labelings of the dual 1-complex with representations and intertwiners. If $\Sigma$ is the boundary of a 3-manifold $M$ with cellular decomposition $\hat{C}$, the partition function $\mathcal{Z}_{\text {sugra }}$ gives rise to a linear map $H_{\Sigma, C} \rightarrow \mathbb{C}$. This map is now independent of how $\hat{C}$ extends the cellular decomposition $C$ into the interior of $M$. Dualizing the vector space $H_{\Sigma, C}$ allows to write the linear map as $\mathbb{C} \rightarrow H_{\Sigma, C}^{*}$. In particular, given a 3-manifold $M$ with boundaries $\Sigma_{1}, \Sigma_{2}$ and cellular decomposition yields a linear map $H_{\Sigma_{1}, C_{1}} \rightarrow H_{\Sigma_{2}, C_{2}}^{*}$, where $C_{1}$ and $C_{2}$ are the induced cellular decompositions of the boundary components. Note that orientation reversal of a 2-manifold corresponds to dualization of the associated vector space (see [4] for details). Thus a given 2-manifold $\Sigma$ with cellular decomposition $C$ defines a linear map $P_{\Sigma, C}: H_{\Sigma, C} \rightarrow H_{\Sigma, C}$ associated with the 3-manifold $\Sigma \times I$ where $I$ is an interval. Here $C$ is extended arbitrarily to the interior of $\Sigma \times I$. Note that $P_{\Sigma, C}$ is a projector due to discretization independence in the interior (see [10] for an explicit construction in the Ponzano-Regge case). The physical state space associated with a 2-manifold $\Sigma$ is defined to be $H_{\Sigma}:=P_{\Sigma, C}\left(H_{\Sigma, C}\right)$. This is independent of the cellular decomposition $C$. Furthermore, $P_{\Sigma, C_{1}}\left(H_{\Sigma, C_{1}}\right)$ and $P_{\Sigma, C_{2}}\left(H_{\Sigma, C_{2}}\right)$ are naturally isomorphic by considering the 3-manifold $\Sigma \times I$ with the two different cellular decompositions $C_{1}$ and $C_{2}$ at the boundaries. The linear map between state spaces for given cellular decompositions descends to a linear map between the physical state spaces $\rho_{M}: H_{\Sigma_{1}} \rightarrow H_{\Sigma_{2}}$.

So far everything seems to be exactly analogous to the case of quantum gravity. In particular, the spin networks on the boundary used to define the state spaces $H_{\Sigma}$ are as usual graphs with nodes and links. Representations are associated with links and intertwiners with nodes. Only now the "group" 
is $\operatorname{OSp}(1 \mid 2)$ and not $\mathrm{SU}(2)$. However, there is a subtlety. Namely, specifying a graph, representations and intertwiners is not enough to define a concrete spin network uniquely. There are ordering ambiguities due to the noncommutativity of the function algebra on $\operatorname{OSp}(1 \mid 2)$. Expressing the labelings (representations and intertwiners) in terms of matrix element functions it is necessary to specify their ordering. A change of ordering introduces factors of -1 for fermionic components. Of course this is not a new degree of freedom and does not affect the abstract definition of the spaces $H_{\Sigma}$. It is rather in concrete calculations that this ambiguity needs to be taken into account. In the circuit diagram formalism this problem is coherently dealt with as the explicit graphical representation takes care of the ambiguities (see [4]). As we shall see this issue resurfaces (as it should) in the discussion of the loop approach.

The standard physical interpretation of the theory is roughly as follows. Consider two 2-manifolds $\Sigma_{1}$ and $\Sigma_{2}$ (thought of as "spacelike hypersurfaces") and a connecting 3-manifold $M$ (thought of as the "time evolution"). Let $\psi_{1}$ and $\psi_{2}$ be elements of the physical state spaces $H_{\Sigma_{1}}$ and $H_{\Sigma_{2}}$. Then the map $\rho_{M}: H_{\Sigma_{1}} \rightarrow H_{\Sigma_{2}}^{*}$ can be evaluated on $\psi_{1}$ and $\psi_{2}$ yielding a complex number. This gives a "transition amplitude" between the state $\psi_{1}$ and the state $\psi_{2}$. There are certain problems with this interpretation however (notably to define a measurement process in this context). It has recently been suggested to consider manifolds with a connected boundary instead and giving a physical interpretation to amplitudes associated with state spaces on such boundaries [33, 34]. This implies in particular, that these boundary surfaces should be thought of as having "timelike components" and that amplitudes are evaluated on a single state. These questions, however, go beyond the framework of the present paper.

\subsection{Canonical quantization}

Similarly to the pure gravity situation, one can develop a loop quantization of supergravity, which provides a canonical framework for the super PonzanoRegge spin foam model. Indeed, the (kinematical) states of a loop quantum supergravity will actually be the osp $(1 \mid 2)$ spin networks which have been identified as boundary states of the super Ponzano-Regge model. From this point of view, the spin foam model defines the space-time version, or path integral, for loop quantum supergravity. It allows to take into account the dynamics of the canonical theory and thus to define a projector onto physical states. The canonical framework is particularly interesting for it introduces geometrical operators such as a (super)length operator, at the kinematical level, which allow to probe the physical meaning of the states of the quantum 
theory.

We can proceed to the canonical analysis of 3-dimensional (Euclidean de Sitter) supergravity on a three-manifold of the type $\mathcal{M}=\mathbb{R} \times \Sigma$. Starting with the super BF action (28), the canonical variables are the (space components of the) supertriad $\mathcal{E}$ and the (space components of the) superconnection $\mathcal{A}$, both valued in the superalgebra osp $(1 \mid 2)$. These variables are conjugate and the Hamiltonian consists of two constraints. The first one, $d_{\mathcal{A}} \mathcal{E}=0$ imposes a vanishing (super)torsion and generates $\operatorname{OSp}(1 \mid 2)$ gauge transformations. We call it the super-Gauss law. The second one, $\mathcal{F}(\mathcal{A})=0$ imposes flatness of the superconnection. It generates "topological" gauge transformations of the supertriad ${ }^{12}$. It can be decomposed into three pieces, a first one generating space diffeomorphism (on $\Sigma$ ), a second one generating right handed supersymmetry transformations and a last one -the Hamiltonian constraint- generating the evolution in time.

One can then "loop quantize" the theory following the same steps as for the usual 3-dimensional gravity theory [21,22]. In this context, one considers the partial observables given by gauge invariant cylindrical functions of the superconnection $\mathcal{A}$. A cylindrical function depends only on the holonomies of $\mathcal{A}$ along the edges of a given closed oriented graph $\Gamma$ :

$$
\phi_{\Gamma}(\mathcal{A})=\phi\left(\left\{U_{e}[\mathcal{A}], e \in \Gamma\right\}\right)
$$

Then one would like to impose $\operatorname{OSp}(1 \mid 2)$ gauge invariance, i.e. invariance under $\operatorname{OSp}(1 \mid 2)$ at the vertices $v$ of the graph $\Gamma$. Heuristically, following the procedures developed in the pure gravity case, this would read as:

$$
\forall k_{v} \in \operatorname{OSp}(1 \mid 2), \phi\left(\left\{k_{s(e)}^{-1} U_{e}[\mathcal{A}] k_{t(e)}, e \in \Gamma\right\}\right)=\phi\left(\left\{U_{e}[\mathcal{A}], e \in \Gamma\right\}\right),
$$

where $s(e)$ and $t(e)$ respectively denote the source and target vertices of an edge $e \in \Gamma$. In fact, a function of the (super)holonomies $U_{e}$ is actually defined as a function of the matrix elements $t\left(U_{e}\right)$ of the (super)group elements. Then, one must be careful since these matrix elements do not commute: we must choose a (full) ordering of all the edges $e$. And the precise definition of gauge invariance will depend on the chosen convention. This is the same problem that one faces when dealing with quantum groups, except the ordering ambiguities only introduce signs in our case, which makes the whole situation (much) simpler.

Using the Haar measure $d \mu$ on $\operatorname{OSp}(1 \mid 2)$ [35], one can introduce the measure product of $d \mu$ on all edges $e \in \Gamma$, which defines a natural scalar product on the space of square integrable cylindrical functions. A basis of

\footnotetext{
${ }^{12}$ The topological transformation reads $\delta \mathcal{E}=d_{\mathcal{A}} \lambda$ for a gauge parameter $\lambda$.
} 
the resulting Hilbert space is provided by the osp $(1 \mid 2)$ super spin networks $[36,37]$. These are labelled by a osp $(1 \mid 2)$ representation for each edge and a $\mathfrak{o} \mathfrak{s p}(1 \mid 2)$ intertwiner for each vertex: $\left|\Gamma, j_{e}, i_{v}\right\rangle$. Let us point that these super spin networks actually depend on the ordering chosen for the edges of the graph. Nevertheless, as the ordering defines the gauge invariance condition, it does not introduce further states in our basis. Let us have a look at an example. We choose a particular ordering of the edges, then a spin network functional will read:

$$
\phi\left(U_{1}, . ., U_{E}\right)=t\left(U_{1}\right) t\left(U_{2}\right) . . t\left(U_{E}\right) \times \text { intertwiners. }
$$

As $\operatorname{OSp}(1 \mid 2)$ gauge invariance is imposed on the intertwiners, if one would like to check how it reads on the edge 1 , then one needs to translate the conditions from the intertwiners to the matrix element $t\left(U_{1}\right)$ commuting it with all the other $t\left(U_{e}\right)$, which introduces signs each time the matrix elements have an odd parity.

Piling up all these Hilbert spaces for each graph $\Gamma$ (i.e summing over graphs $\Gamma$ ), one constructs a Hilbert space of $\mathrm{OSp}(1 \mid 2)$ invariant states of the superconnection, which can be considered as the Hilbert space of square integrable functions over gauge equivalence classes of (generalized) superconnections.

One last remark on $\mathfrak{o s p}(1 \mid 2)$ spin networks is to point out that, as all $R^{j}$ representations are symmetric/antisymmetric tensor products of the fundamental $R^{1 / 2}$ representation, one can decompose any super spin network into a superposition of fundamental loops -labelled by $R^{1 / 2}$ - by splitting each $R^{j}$ labelled edge into a symmetrized/antisymmetrized system of ropes, each rope corresponding to a $R^{1 / 2}$ representation [37].

To finish the quantization, one needs to impose the flatness constraint $\mathcal{F}(\mathcal{A})=0$. First, one gets diffeomorphism invariant states by considering equivalence classes of graphs under diffeomorphisms of $\Sigma$. Then, one would like to implement the (right handed) supersymmetry constraint and the Hamiltonian constraint on the resulting Hilbert space. In the present work, we are not going to discuss this issue directly within the canonical framework. Instead, we tackle this issue from the spin foam point of view: we construct the path integral for the theory, which, once interpreted within the canonical framework, provides us with a projector onto the physical Hilbert space (of states satisfying all the constraints). Indeed, the super Ponzano-Regge model, implementing the path integral of the super BF action (28), is a topological state sum whose boundary states (kinematical states) are actually the $\mathfrak{o s p}(1 \mid 2)$ spin networks and which defines a projector on the physical states of supergravity (see Section 5.3). 
An attractive aspect of the Hilbert space of (super) spin networks is its geometrical interpretation. Indeed, adapting techniques of $2+1$-dimensional loop quantum gravity [22] just like it is possible to extend results of $(3+1)$ loop quantum gravity to loop quantum supergravity [36], super spin networks appear as eigenvectors of the super length operator. More precisely, the super length of a curve $c: \tau \in[0,1] \rightarrow c(\tau) \in \Sigma$ is:

$$
L_{c}=\int_{[0,1]} d \tau \sqrt{\dot{c}^{a}(\tau) \dot{c}^{b}(\tau) S T r_{o s p(1 \mid 2)}\left(\mathcal{E}_{a}(c(\tau)) \mathcal{E}_{b}(c(\tau))\right)} .
$$

One can quantize it as an operator $\widehat{L_{c}}$ by replacing $\mathcal{E}$ by the derivative with respect to $\mathcal{A}$ (and regularizing the expression) just as the gravity case. Then its action is diagonal on super spin networks. The eigenvalue depends on the $\mathfrak{o s p}(1 \mid 2)$ representations living on the edges of the spin network intersected by $c$ and is given by the sum of the squareroot of the Casimir of these representations:

$$
\widehat{L_{c}}\left|\Gamma, j_{e}, i_{v}\right\rangle=\left(\sum_{e \mid e \cap c \neq \emptyset} \sqrt{j_{e}\left(j_{e}+\frac{1}{2}\right)}\right)\left|\Gamma, j_{e}, i_{v}\right\rangle .
$$

One should be careful that this super length operators depends on both the metric field and the fermionic fields. It is not the simple length operator $\widehat{l_{c}}$ :

$$
l_{c}=\int_{[0,1]} d \tau \sqrt{\dot{c}^{a}(\tau) \dot{c}^{b}(\tau) \operatorname{Tr}_{s u(2)}\left(e_{a}(c(\tau)) e_{b}(c(\tau))\right)} .
$$

Indeed, $l_{c}$ is invariant under $\mathrm{SU}(2)$ but not under $\operatorname{OSp}(1 \mid 2)$, so that its action cannot be implemented on a space of $\mathrm{OSp}(1 \mid 2)$ invariant functionals. Considering a small curve intersecting a single edge $e$ labelled with the representations $R^{j}, \widehat{l_{c}}$ would be the square root of the $\mathrm{SU}(2)$ Casimir operator $J^{i} J^{i}$ on $R^{j}$ and would distinguish the two isospins $V^{j}$ and $V^{j-1 / 2}$ assigning them different length values $\sqrt{j(j+1)}$ and $\sqrt{\left(j-\frac{1}{2}\right)\left(j+\frac{1}{2}\right)}$. Then the fermionic fields contribute $-\frac{j}{2}$ (resp. $\left.\frac{1}{2}\left(j+\frac{1}{2}\right)\right)$ to the Casimir on $V^{j}$ (resp. $\left.V^{j-1 / 2}\right)$, so that the super length is diagonal.

From this perspective, one can view super spin networks as supersymmetric superposition of (pure) geometry states given by the usual $\mathfrak{s u}(2)$ spin networks (labelled with $\mathfrak{s u}(2)$ representations $V^{j}$ ).

\subsection{Identifying Fermions on the spin foam}

As the Ponzano-Regge model provides us with a path integral formulation for three-dimensional quantum gravity, the super Ponzano-Regge model based 
on $\mathfrak{o s p}(1 \mid 2)$ provides us with a path integral for three-dimensional quantum supergravity. In this context, it would be interesting to distinguish the gravity degrees of freedom from the fermionic degrees of freedom in our supersymmetric state sum model. In particular, we know that fermions live at the nodes of spin networks in standard loop quantum gravity [38]. However, in the present super Ponzano-Regge model the fermionic field is taken into account in the holonomies of the super connection $\mathcal{A}$ and therefore in the representations (of $\mathfrak{o s p}(1 \mid 2)$ ) living on the links of the super spin networks. It would be enlightening to reformulate the super Ponzano-Regge model with "fermions" at the nodes in such a way as to make the link with the usual loop quantum gravity formalism.

In the following, we propose such a picture in which we can identify objects similar to fermion worldlines on the spin foam. It looks like some fermions propagating on some gravity background and therefore the super Ponzano-Regge model appears as a path integral for gravity plus fermions. Nevertheless, we do not prove that the proposed picture is "right", in the sense that the paths drawn on the spin foam truly correspond to the fermionic degrees of freedom. To verify this conjecture one would have to to study a continuum limit (through asymptotics of the supersymmetric $6 j$-symbols), or a semi-classical limit, and we postpone this analysis to future work.

Let us start by summarizing the geometrical structure of the (super) Ponzano-Regge model. It is usually formulated on a simplicial triangulation of a three-dimensional manifold: we work with tetrahedra glued along common triangles. We can extend this to a generic cellular decomposition made of 0-cells (points), 1-cells (lines), 2-cells and 3-cells. In the spin foam context, we consider the dual structures:

$\begin{array}{ll}\text { spinfoam } & \leftrightarrow \text { triangulation } \\ \text { vertices } & \leftrightarrow 3-\text { cells } \\ \text { edges } & \leftrightarrow 2-\text { cells } \\ \text { faces } & \leftrightarrow 1-\text { cells } \\ \text { volumes } & \leftrightarrow 0-\text { cells }\end{array}$

so that vertices are linked by edges corresponding to the 3-cells glued along 2-cells. Usually, we consider only the dual 2-complex, meaning that we forget about the last line of the above correspondence, "losing" the notion of dual volumes (and therefore of points in the triangulation).

Now, in the state sum model, we attach (super) group representations to the 1-cells of the triangulation and intertwiners to the 2-cells (intertwining the representations living on its boundary edges). In the spin foam setting, representations are attached to faces and intertwiners live on the edges (intertwining the representations of the faces incident on a given edge). This 


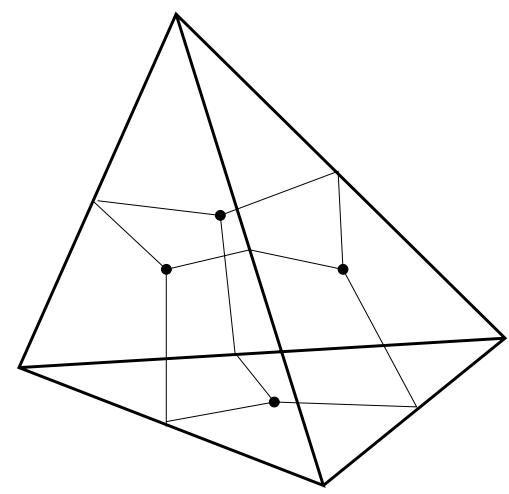

Figure 8: Tetrahedron and its boundary spin network.

way, taking a generic slice of the spin foam, we obtain a graph with representations on its links (cut faces) and intertwiners at its nodes (cut edges), i.e. a spin network.

Now the amplitude of a spin foam configuration is the product of amplitudes assigned to each vertex (3-cell) multiplied with weights associated to the edges (2-cells) and faces (1-cells). The weight of a face is the (super) dimension of the representation. The amplitude of a vertex is given by the evaluation of its boundary spin network. More precisely, we consider the 3cell around a vertex. Its boundary is a sphere (consisting of several 2-cells). The intersections of the sphere with the faces around the vertex form a (super) spin network (on the sphere). This boundary spin network can also be seen as the 1-skeleton of the dual complex of the boundary of the 3-cell. In the case of a simplicial decomposition, the 3-cells are the tetrahedra and the boundary spin networks can be identified with the $6 j$-symbols (see Figure 8 ).

In the following, we will restrict our analysis to 3-valent spin networks, i.e. the 2-cells of the triangulation are all triangles. As it is straightforward to decompose an $n$-valent intertwiner into 3 -valent intertwiners (the same way that a generic polygon can be decomposed into internal triangles), the whole construction will be easily extended to the generic case.

Let us forget about sign ambiguities for the moment and analyze the structure of $\mathfrak{o s p}(1 \mid 2)$ spin networks. Comparing to the Ponzano-Regge model, we expect pure gravity degrees of freedom to be encoded in $\mathfrak{s u}(2)$ structures. It is then natural to decompose each osp $(1 \mid 2)$ representation into its $\mathfrak{s u}(2)$ isospins. Applying this decomposition to an $\mathfrak{o s p}(1 \mid 2)$ spin network, we must distinguish two types of $\mathfrak{o s p}(1 \mid 2)$ intertwiners: 
(i) Intertwiners intertwining representations $R^{j_{1}}, R^{j_{2}}, R^{j_{3}}$ with $j_{1}+j_{2}+$ $j_{3} \in \mathbb{N}$, in which case there exists a component intertwining between the isospins $V^{j_{1}}, V^{j_{2}}, V^{j_{3}}$;

(ii) Intertwiners intertwining representations $R^{j_{1}}, R^{j_{2}}, R^{j_{3}}$ with $j_{1}+j_{2}+$ $j_{3} \in \mathbb{N}+\frac{1}{2}$, in which case there doesn't exist any $\mathfrak{s u}(2)$ intertwiner between $V^{j_{1}}, V^{j_{2}}$ and $V^{j_{3}}$.

It appears natural to call an intertwiner of the type (i) a bosonic intertwiner, or boson, and an intertwiner of the type (ii) a fermionic intertwiner, or fermion.

To analyze the structure of an $\mathfrak{o s p}(1 \mid 2)$ spin network, let us introduce a graphical convention for the $\mathfrak{s u}(2)$ spin networks resulting from the decomposition of the $\mathfrak{o s p}(1 \mid 2)$ spin network: solid and dotted lines corresponding respectively to upper and lower isospins, $V^{j}$ or $V^{j-\frac{1}{2}}$, of the original $R^{j}$ representation.

Proposition 5.1. Considering a 3-valent osp $(1 \mid 2)$ spin network, it contains an even number of fermionic intertwiners (fermions) and of bosonic intertwiners (bosons). Decomposing each osp $(1 \mid 2)$ representation into its two $\mathfrak{s u}(2)$ isospins, an upper one and a lower one, the osp $(1 \mid 2)$ spin network appears as a sum of $\mathfrak{s u}(2)$ spin networks, which we draw with solid and dotted links corresponding to upper and lower $\mathfrak{s u}(2)$ representations. Then, in each of the resulting $\mathfrak{s u}(2)$ spin networks, dotted links form lines on the spin network linking the fermions: the set of dotted lines can be partitioned into lines between pairs of fermions (each fermion being linked to a single other fermion) and closed loops.

Let us first point out that a 3 -valent graph has an even number of nodes ${ }^{13}$. The key point of the proof is that there is an even number ( 0 or 2$)$ of dotted lines at a bosonic intertwiner and an odd number (1 or 3 ) at a fermionic intertwiner (as drawn in Figure 6). Then, from the point of view of the dotted lines, we can forget the solid lines and the bosonic intertwiners, and only consider the graph made of the dotted links and the fermionic intertwiners, which we call the dotted graph.

Among these fermionic intertwiners, there are some which are attached to only one dotted line. Such a fermion $f_{1}$ is linked directly to another fermion $f_{2}$ through that dotted line. Thus, we can put aside that couple of fermions, $f_{1}$ and $f_{2}$, and that dotted line. The dotted graph left after removing these two fermions is still a graph of the same type, since $f_{2}$ had

\footnotetext{
${ }^{13}$ If $E$ is the number of links of the graph and $V$ its number of nodes then $2 E=3 V$.
} 


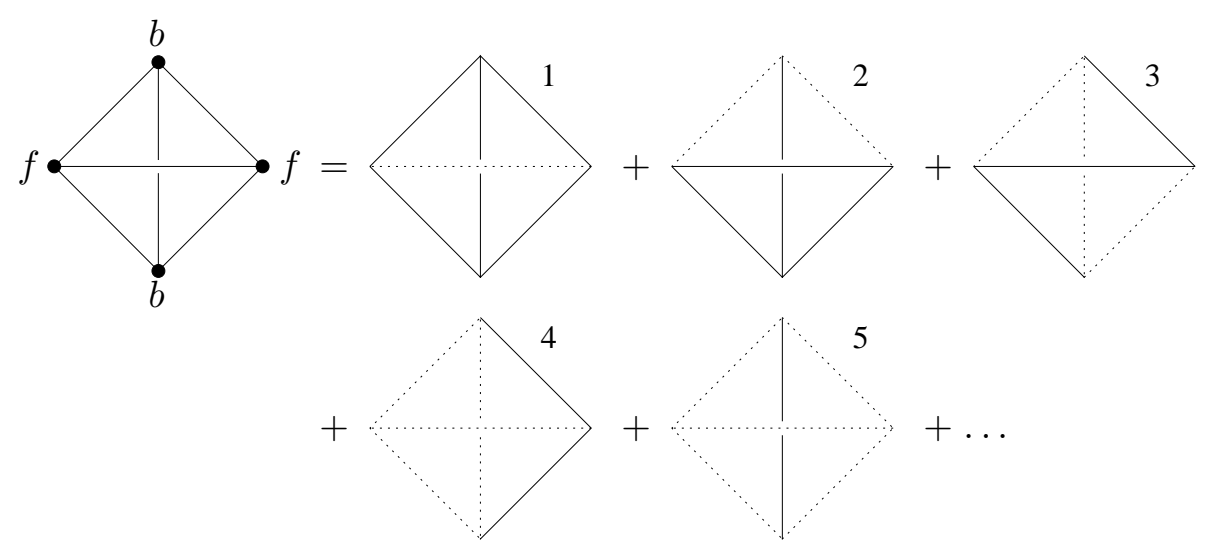

Figure 9: Decomposition of the tetrahedral net with two fermionic intertwiners on $\mathfrak{s u}(2)$ spin networks with solid/dotted lines for upper/lower $\mathfrak{s u}(2)$ isospins (the dots mean summing over the symmetric situations). One sees that the dotted links form a path between the two fermions, plus possibly a loop. Also, one notices, in the case of the diagram 5 for example, that the choice path/loop is not unique and that one can even forget the loop and consider a single long dotted line linking the two fermions.

either one attached dotted link (the one linking it to $f_{1}$ ) or three dotted links in which case $f_{2}$ becomes a bivalent node on the dotted graph and we can forget it. After having removed all such fermions, either there are none left and the dotted graph is a set of loops, or we are left with a 3-valent graph, which we call the reduced dotted graph.

Then any 3-valent graph has an even number of nodes and it is moreover possible to show by induction that we can decompose it into lines connecting pairs of vertices and (closed) loops.

Let us point out that the decomposition of the dotted lines -linking pairs of fermions- and loops is not unique at all. Therefore, there is a choice of "interpretation" in the choice of which fermion is linked to which other one. We illustrate these considerations with the example of the tetrahedron net $(6 j$-symbol) in Figure 9 and Figure 10.

Now, let us come back to the spin foam model. An $\mathfrak{o s p}(1 \mid 2)$ spin foam is labelled by $\mathfrak{o s p}(1 \mid 2)$ representations on its faces, and the intertwiners live on its edges, which are then either bosonic or fermionic. The amplitude of a given $\mathfrak{o s p}(1 \mid 2)$ spin foam is the product of the boundary spin networks corresponding to its 3-cells/dual vertices. Each of these boundary spin networks is the sum of many $\mathfrak{s u}(2)$ spin networks on which are drawn loops and 


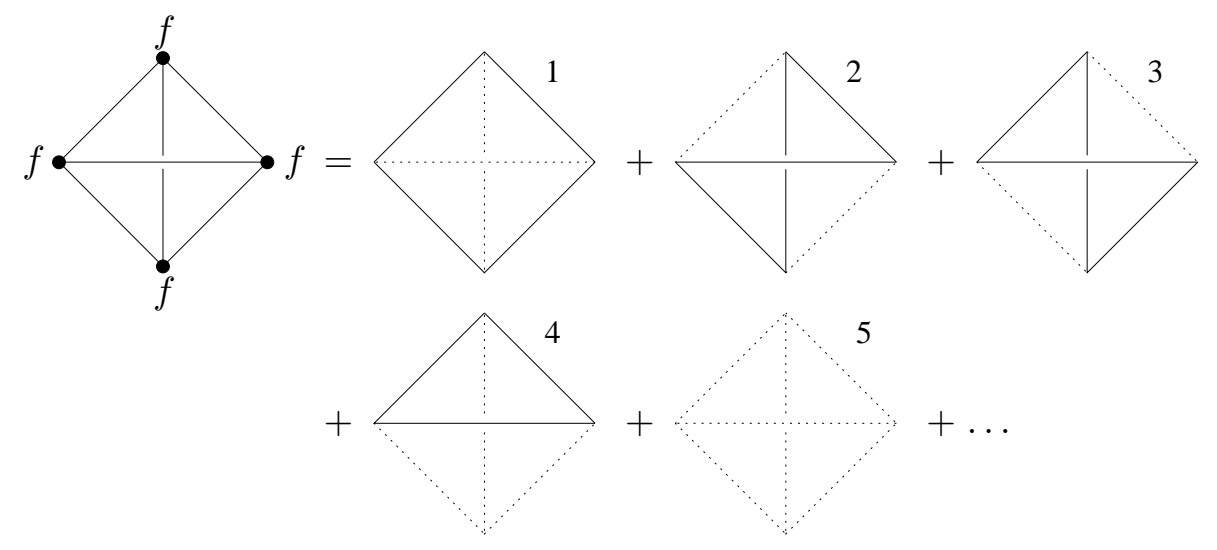

Figure 10: Decomposition of the tetrahedral net with four fermions: The dotted links form two lines, each one linking two fermions together. In the diagrams $1,2,3$, it is straightforward to see which fermion is linked with which other. In the diagrams 4,5, the situation is more complicated and it is just a question of interpretation to decide which fermionic intertwiner is linked with which other one, i.e. which fermion goes where.

lines linking each fermionic intertwiner to another one. Let us emphasize that each edge corresponds to a cable that we cut into two osp (1|2) intertwiners which are the same (up to some sign conventions), so that both are fermionic or both are bosonic. This property is inherited by the $\mathfrak{s u}(2)$ intertwiners, drawn with solid and dotted lines, when decomposing the osp $(1 \mid 2)$ representations into $\mathfrak{s u}(2)$ isospins.

The resulting space-time picture is that we have fermions propagating on the edges of the spin foam and meeting at the vertices. Each term of the sum describes what happens at the vertices (fermion scattering,...) and therefore can be understood as a Feynman diagram for fermions drawn on the geometrical skeleton of the spin foam. At the level of the amplitude, the full amplitude is the sum of the amplitudes of $\mathfrak{s u}(2)$ spin foams on which are drawn fermionic loops and lines.

On the triangulation, intertwiners live on the triangles, which are thus fermionic or bosonic. Then, each term of the boundary spin network of a given 3-cell tells one which fermion (fermionic triangle) goes where. And one can build a sequence of triangles by following a given fermionic triangle through every 3-cell, simply the path of a fermion.

Through this construction, we have identified fermionic degrees of freedom attached to the intertwiners, which matches with the canonical loop 
quantum gravity picture. Moreover, we have interpreted the super PonzanoRegge model as providing a path integral for gravity+fermions, in which the space-time is described as fermions propagating on a "background" geometry described with the usual $\mathfrak{s u}(2)$ representations of the Ponzano-Regge model. Let us point out that it is not simply a background geometry since the fermions modify the geometrical properties -the length of curves for example- along their path.

To sum up, given a triangulated three-dimensional manifold $\Delta$, one labels its 1-cells with $\mathfrak{o s p}(1 \mid 2)$ representations and assigns an amplitude to each configuration (consistent labelling under the recoupling theory of $\mathfrak{o s p}(1 \mid 2)$ ). Then the full super Ponzano-Regge partition function for $\Delta$ is the sum of these amplitudes over all configurations. Considering a given term (fixed $\mathfrak{o s p}(1 \mid 2)$ labels), decomposing each $\mathfrak{o s p}(1 \mid 2)$ representations into $\mathfrak{s u}(2)$ representations, the amplitude appears like a (supersymmetric) superposition of pure gravity/geometry space-time states/configurations defined by $\mathfrak{s u}(2)$ edge labels like in the Ponzano-Regge model. This superposition of gravity states can also be interpreted as one pure gravity state plus gravity states with propagating fermions.

This can be written as a decompositions of the partition function for a fixed closed triangulated manifold $\Delta$ :

$$
Z(\Delta)=\sum_{J_{\mathfrak{o s p}(1 \mid 2)}} Z_{(S P R)}(\Delta, J)=\sum_{J_{\mathfrak{o s p}(1 \mid 2)}} \sum_{j_{\mathfrak{s u}(2)} \hookrightarrow J} c(J, j) Z_{(\mathfrak{s u}(2))}(\Delta, j) .
$$

$Z_{(S P R)}$ is the super Ponzano-Regge amplitude for specified colorings $J_{\mathfrak{o s p}(1 \mid 2)}$. When we decompose osp $(1 \mid 2)$ representations into $\mathfrak{s u}(2)$ isospins, this amplitude reads as a sum of $\mathfrak{s u}(2)$ terms, with $c(J, j)$ some normalization coefficients (such that the sum is supersymmetric). Each term $Z_{(\mathfrak{s u}(2))}(\Delta, j)$ is a product of 6 j-symbols of $\mathfrak{s u}(2)$ and the coefficients $c(J, j)$ can be considered as part of the measure for the sum. Then, using our graphical notation with solid/dotted lines, we can identify the fermions, living on triangles, and the fermionic lines corresponding to each term $c(J, j) Z^{(P R)}(\Delta, j)$ and it turns out that a term is non-vanishing iff the fermionic lines form loops on the spin foam. Therefore, one could swap the sums on $\mathfrak{o s p}(1 \mid 2)$ and $\mathfrak{s u}(2)$ labels in the partition function. This means first summing on $\mathfrak{s u}(2)$ representation labels and then on the possible consistent ways of embedding them into $\mathfrak{o} \mathfrak{s p}(1 \mid 2)$ representations, i.e. over consistent choices of solid/dotted lines in the evaluation of the 3-cell boundary spin networks. These consistent configurations will then be defined by all possible sets of (closed) loops, the fermionic paths, drawn on the spin foam. Let us emphasize that the sum over $\mathfrak{s u}(2)$ is a generalised one: we do not sum over one representation label $j$ for each face of the spin foam (like in the Ponzano-Regge model) but we sum 
over couples of labels $-j$ and $j-\frac{1}{2}$ - for each face and allow different labels $-j$ or $j-\frac{1}{2}$ - around a single face, one for each adjacent vertex (equivalently, at the level of the triangulation, a representation $j$ is attached to a 1-cell and we sum over assignements of $j$ or $j-\frac{1}{2}$ to each 3 -cell adjacent to that 1-cell). Then, when $\Delta$ has boundaries, one must first check which boundary nodes are fermionic and then the "loops" must also include lines drawn between pairs of boundary fermions. At the end of the day, the partition function looks like a sum over gravity configurations and fermion paths drawn on the spin foam, or equivalently gravity+fermions Feynman diagrams.

Finally, we emphasize that the whole construction is just a "nice" picture for the moment and that we have not provided a proof that the fermionic intertwiners are truly physical fermions and that the dotted lines are truly fermion paths. It is true that this interpretation seems natural from the point of view of the representation theory and that it leads to an interesting understanding of the partition function as a sum over coupled gravity + fermions configurations. Nevertheless, only a semi-classical analysis or the study of a continuum limit could settle the issue and prove the picture right or wrong.

\section{6 q-deformation: $U_{q}(\mathfrak{o s p}(1 \mid 2))$ spin foams}

In spin foam models for quantum gravity, quantum deforming the gauge group has two uses. On the one hand, it regularizes the state sum by making finite the sum over representations. On the other hand, it is supposed to take into account a positive cosmological constant. In the case of the PonzanoRegge model, quantum deforming $\mathrm{SU}(2)$ into $\mathrm{SU}_{q}(2)$ leads to the TuraevViro model [24]. Choosing $q=e^{\frac{2 \pi i}{N}}$ to be a root of unity reduces the number of inequivalent irreducible representations to be finite with a spin cutoff $j \leq(N-2) / 2$ : Having finite sums makes the Turaev-Viro model welldefined. Let us recall the q-dimensions of the representations, which enter the state sum instead of the ordinary dimensions:

$$
\operatorname{qdim}(j)=\frac{q^{j+\frac{1}{2}}-q^{-j-\frac{1}{2}}}{q^{\frac{1}{2}}-q^{-\frac{1}{2}}}=\frac{\sin \left((2 j+1) \frac{\pi}{N}\right)}{\sin \left(\frac{\pi}{N}\right)} \underset{q \rightarrow 1}{\longrightarrow} 2 j+1 .
$$

Moreover, it has been shown that the cosmological constant $\Lambda$ is linked with the parameter $q$ through $N \sqrt{\Lambda}=2 \pi$. Mathematically, this comes from the reformulation of three-dimensional quantum gravity as the sum of two $\mathrm{SU}(2)$ Chern-Simons theories. More precisely, the partition function of the TuraevViro model, as the quantization of BF theory with cosmological constant, appears to be the squared absolute value of the Chern-Simons amplitude (with level $N$ ) [39]. On a more empirical level, it can be seen from the 
asymptotics of the quantum $6 j$-symbols which turns out to be the cosine of the Regge action for (discretized) gravity with cosmological constant.

In this context, it is very appealing to q-deform the super Ponzano-Regge model writing a "super Turaev-Viro" model based on the quantum group $U_{q}(\mathfrak{o s p}(1 \mid 2))$. This is a very simple quantum (super)group. Just like for all the $U_{q}(\mathfrak{o s p}(1 \mid 2 n))$, there exists an invariant Haar measure and a Peter-Weyl decomposition [40, 41]. Tensor products of irreducible representations are totally reducible, so that one can straightforwardly write a corresponding topological model using the usual techniques to deal with quantum groups. The partition function can formally be written as (40), except with the $6 \mathrm{j}$ symbols replaced by q-deformed $6 \mathrm{j}$-symbols. However, this expression is formal as explained in Section 5.2 and the "real" definition is through generalized circuit diagrams [4]. The proof of topological invariance generalizes to this setting [23].

Then, for $q=e^{\frac{2 \pi i}{N}}$ an $N$ th root of the unity, there is once again a finite number of irreducible representations $R^{j, \pm}$ labelled by half-integers $0 \leq j \leq(N-1) / 2$ and a parity \pm . Choosing, as we did for $\mathfrak{o s p}(1 \mid 2)$, the parity to be the physical one (i.e. to obey the spin-statistics relation) their q-superdimension is:

$$
\mathrm{q} \operatorname{Sdim}\left(R^{j}\right)=(-1)^{2 j} \frac{q^{2 j+1}+q^{-2 j}}{q+1}=(-1)^{2 j} \frac{\cos \left((4 j+1) \frac{\pi}{N}\right)}{\cos \left(\frac{\pi}{N}\right)} \underset{q \rightarrow 1}{\longrightarrow}(-1)^{2 j} .
$$

Therefore, in this setting, sums over representations are finite and the resulting state sum is well-defined. For more details on the representation theory and more particularly on the tensor products of representations, we refer to $[40,41]$.

Paralleling the situation for gravity, three-dimensional (Euclidean de Sitter) supergravity can be formulated as the sum of two $\operatorname{OSp}(1 \mid 2)$ ChernSimons theories, as explained in Section 4.1. Thus, its quantization should yield the quantum group $U_{q}(\mathfrak{o s p}(1 \mid 2))$ with $q$ related to the cosmological constant as above. Furthermore, the relation between quantized Chern-Simons theory and the Turaev-Viro model is the same for super (quantum) groups as for ordinary (quantum) groups. Thus, the super Turaev-Viro model should indeed encode the quantization of the supersymmetric theory with cosmological constant. It would be very interesting to work out the asymptotics of the super quantum $6 j$-symbols to check the relation $q \leftrightarrow \Lambda$ on a semi-classical level.

Let us point out a weakness in the link between the q-deformation and the cosmological constant: the $q$-deformation does not appear naturally in 
the loop quantum gravity setting. Indeed, the "kinematical states" of quantum gravity are $\mathrm{SU}(2)$ spin networks and the cosmological constant $\Lambda$ appears only in the Hamiltonian constraint governing the dynamics. The same occurs for supergravity. Through the q-deformation of the Ponzano-Regge model into Turaev-Viro model, it seems that, at the spin foam level, the $q$-deformation allows to take into account properly the dynamics implied by the cosmological constant. We expect the same situation in supergravity. Still, it would be very interesting to study how the $q \leftrightarrow \Lambda$ relation could appear in the canonical loop gravity framework, both in the gravity case and in the supergravity case.

\subsection{Towards a Lorentzian model: $\mathfrak{o s p}_{L}(1 \mid 2)$ spin foams}

Up to now, we have dealt with the Euclidean Ponzano-Regge model corresponding to a quantization of Euclidean (or Riemannian) gravity. We can also study the Lorentzian version $[42,43]$ quantizing $2+1$-dimensional gravity and extend to a supersymmetric Lorentzian Ponzano-Regge model which would be a state sum for Anti-de Sitter supergravity in $2+1$ dimensions.

Let us start by describing the Lorentzian Ponzano-Regge model. As the gauge group (in the connection formalism) of $2+1$-dimensional gravity is $\mathrm{SO}(2,1)$ instead of $\mathrm{SO}(3)$, we build a model invariant under $\mathrm{SU}(1,1)$ instead of under $\mathrm{SU}(2)$. It uses the unitary irreducible representations of $\mathfrak{s u}(1,1)$, which are infinite dimensional. These representations are of two kinds (restricting ourselves to the principal representations, appearing in the Plancherel formula for $\mathfrak{s u}(1,1)$ ): a continuous series and two discrete series, one negative and one positive. The representation spaces are similar to the $\mathfrak{s u}(2)$ case, with a basis $|m\rangle$ labelled by half-integers. The representations of the continuous series have a basis labelled by all $m \in \mathbb{Z}$ (or $m \in \mathbb{Z}+1 / 2$ depending on their parity) whereas the representations of the discrete series are in a way complementary to the $\mathfrak{s u}(2)$ representations and have a basis spanned by a bounded label, $m \geq j$ (for the positive representation $\left.V_{(\mathfrak{s u}(1,1))}^{j}\right)$ or by $m \leq-j$ (for the negative series). The difference between $\mathfrak{s u}(2)$ and $\mathfrak{s u}(1,1)$ can be understood on the Lie algebra level as arising from a difference in the hermiticity conditions: For $J_{ \pm}$defined in $(32), \mathfrak{s u}(2)$ is characterized by $J_{ \pm}^{\dagger}=J_{\mp}$ whereas $\mathfrak{s u}(1,1)$ is defined by $J_{ \pm}^{\dagger}=-J_{\mp}$ (for more details, see for example [22]).

Then, these $\mathfrak{s u}(1,1)$ representations label the edges of the triangulation (or the faces of the spin foam) and have a clear geometric interpretation: the representations of the continuous series correspond to space-like edges and the representations of the positive (resp. negative) discrete series to 
future (resp. past) oriented time-like edges. Following [43], one can then write a topological model based on the whole category of continuous and discrete representations. However, one can also build a "restricted" topological model using solely the positive discrete series (because this set of representations is closed under tensor product). This "restricted" model is particularly interesting for it contains only oriented time-like representations (corresponding to time-like edges) and is interpreted using time-like bound$\operatorname{aries}^{14}$. The geometric interpretation is given by assigning a length $j-\frac{1}{2}$ to an edge labelled with the representation $V_{(\mathfrak{s u}(1,1))}^{j}$. This is supported by many arguments, similar to the Euclidean case (Ponzano-Regge model), both from the representation theory of $\mathfrak{s u}(1,1)[42,43]$ and from the canonical theory (loop quantum gravity) [22]. This way, we have a natural (Lorentzian) model with quantized proper time.

Now, we would like to build a supersymmetric Lorentzian Ponzano-Regge model. Following section 4, it would correspond classically to Anti-de Sitter (Lorentzian) gravity, using the gauge group $\operatorname{OSp}_{L}(1 \mid 2)$, the supersymmetric extension of $\mathrm{SU}(1,1)$, instead of $\operatorname{OSp}_{E}(1 \mid 2)$. We would only extend supersymmetrically the (positive) discrete series of representations and give a supersymmetric version of the restricted Lorentzian model. Its natural interpretation would be with time-like boundaries and fermions on these time-like boundaries.

Mimicking the representation theory of $\mathfrak{o s p}_{E}(1 \mid 2)$ (choosing weights $m \geq$ $j$ instead of $|m| \leq j$ and rotating by $i$ the generators $J \pm$ ), we find representations $R^{j}$ of $\mathfrak{o s p}_{L}(1 \mid 2)$ which are sums of two (unitary, infinite dimensional) representations, $V^{j}$ and $V^{j-\frac{1}{2}}$, of $\mathfrak{s u}(1,1)$. Each $V^{k}$ representation can be written in a basis similar to the $\mathfrak{s u}(2)$ case $|k, m\rangle$, but with weights $m \geq k$.

\footnotetext{
${ }^{14}$ In the framework of the restricted model, there is a natural light cone structure on the spin foam. Indeed, considering a point in the triangulated manifold, the set of its future events is given by all points linked to it by a sequence of future oriented edges. In this context, one can easily identify the double cones (or diamonds) and study the corresponding observables. This brings the model close to the framework of algebraic quantum field theory.
} 
The explicit action of the generators is:

$$
\begin{aligned}
J_{3}|j, j, m\rangle & =m|j, j, m\rangle \\
J_{3}\left|j, j-\frac{1}{2}, m\right\rangle & =m\left|j, j-\frac{1}{2}, m\right\rangle \\
J_{ \pm}|j, j, m\rangle & =(m(m \pm 1)-j(j+1))^{1 / 2}|j, j, m \pm 1\rangle \\
J_{ \pm}\left|j, j-\frac{1}{2}, m\right\rangle & =\left(m(m \pm 1)-\left(j-\frac{1}{2}\right)\left(j+\frac{1}{2}\right)\right)^{1 / 2}\left|j, j-\frac{1}{2}, m \pm 1\right\rangle, \\
Q_{+}|j, j, m\rangle & =-(m-j)^{1 / 2}\left|j, j-\frac{1}{2}, m+\frac{1}{2}\right\rangle \\
Q_{+}\left|j, j-\frac{1}{2}, m\right\rangle & =\frac{i}{2}\left(m+j+\frac{1}{2}\right)^{1 / 2}\left|j, j, m \pm \frac{1}{2}\right\rangle \\
Q_{-}|j, j, m\rangle & =-i(m+j)^{1 / 2}\left|j, j-\frac{1}{2}, m-\frac{1}{2}\right\rangle, \\
Q_{-}\left|j, j-\frac{1}{2}, m\right\rangle & =-\frac{1}{2}\left(m-\left(j+\frac{1}{2}\right)\right)^{1 / 2}\left|j, j, m-\frac{1}{2}\right\rangle .
\end{aligned}
$$

The commutation relations are:

$$
\begin{array}{rrrl}
{\left[J_{3}, J_{ \pm}\right]= \pm J_{ \pm}} & {\left[J_{+}, J_{-}\right]=-2 J_{3}} \\
{\left[J_{3}, Q_{ \pm}\right]= \pm \frac{1}{2} Q_{ \pm} \quad\left[J_{ \pm}, Q_{ \pm}\right]=0} & {\left[J_{ \pm}, Q_{\mp}\right]=-i Q_{ \pm}} \\
\left\{Q_{ \pm}, Q_{ \pm}\right\}=\mp \frac{i}{2} J_{ \pm} & \left\{Q_{\mp}, Q_{ \pm}\right\}=\frac{1}{2} J_{3} .
\end{array}
$$

The "hermiticity" relations are $J_{3}^{\dagger}=J_{3}, J_{ \pm}^{\dagger}=J_{\mp}$ and $Q_{+}^{\dagger}= \pm Q_{-}, Q_{-}^{\dagger}=$ $\mp Q_{+}$, the sign reflecting the parity assigned to the representation just as in the $\mathfrak{o s p}_{E}(1 \mid 2)$ case.

The (3-valent) intertwiners can be defined through the decomposition of the tensor product of two representations. In the $\mathfrak{s u}(1,1)$ case, it reads as:

$$
V^{j} \otimes V^{k}=\bigoplus_{l \geq j+k} V^{l}
$$

where $l$ goes by integer steps. The condition $l \geq j+k$ is the anti-triangular inequalities for a triangle with only timelike edges. We get the same structures for the supersymmetric extension:

$$
R^{j} \otimes R^{k}=\bigoplus_{l \geq j+k} R^{l}
$$

where $l$ now goes through all half-integer values. 
We are not going to describe the details of explicitly constructing the Lorentzian super-Ponzano-Regge model. Just as for the Lorentzian PonzanoRegge model, we would need to tackle the difficulties linked with the use of a non-compact group and infinite dimensional representations. This is beyond the scope of the present paper. Nevertheless, we already see by the similarity of the representation theories that all the considerations made for the Euclidean case will translate to the Lorentzian case: we can also distinguish fermionic and bosonic intertwiners in the Lorentzian model and a Lorentzian super-Ponzano-Regge state sum will have a natural interpretation as a spin foam model with fermions on timelike boundaries.

\section{Conclusions and Outlook}

We have presented a general diagrammatic formalism to deal with supersymmetric spin foam models. It is on the one hand a special case of the formalism presented in [4] and on the other hand an extension to the graded case of the connection formulation of spin foam models. Using the formulation of three-dimensional supergravity as a topological BF theory with an $\operatorname{OSp}(1 \mid 2)$ gauge group, we have defined a super-Ponzano-Regge model, a discretized path integral for quantum supergravity. The model, extension of the original Ponzano-Regge model, is naturally independent of the discretization. We have described the extension of the model to a topological quantum field theory, thus providing a description of all the elemets of the quantum theory of supergravity in dimension three. We have also studied aspects of canonical loop quantum supergravity, expressed its kinematical states as super spin networks and considered its relation to our super-Ponzano-Regge model.

Since supergravity contains fermionic degrees of freedom, we have considered the problem of identifying these in the super-Ponzano-Regge model. It turned out that $\mathfrak{o s p}(1 \mid 2)$ intertwiners are of two kinds, which we tentatively called "bosonic" and "fermionic". Starting with this assumption, we can identify (closed) fermion paths on the spin foam. We can also write the partition function of the supersymmetric model as a sum over (supersymmetric) superpositions of pure gravity/geometry states, which we suggest to interpret as a sum over gravity configurations plus fermion paths drawn onto some background geometry (defined by a pure gravity state).

We would need to study in more details how to extend the present procedures to the Lorentzian case, therefore studying (Anti-de Sitter) Lorentzian quantum supergravity in $2+1$ dimensions. The (restricted) $\mathfrak{o s p}_{L}(1 \mid 2)$ model, using solely time-like representations, describes time-like boundaries with 
fermions on these boundaries. One should define properly this model, but also see if one could extend supersymmetrically the continuous series of representations of $\mathfrak{s u}(1,1)$ in order to include space-like edges in the model.

It could be also interesting to look at theories with higher supersymmetries, e.g. invariant under $\operatorname{OSp}(p \mid 2) \times \operatorname{OSp}(q \mid 2)$ [11]. As long as the theory can be defined as a kind of BF theory there does not seem to be any obstacle.

One of the most intriguing further directions is certainly the generalization to higher dimensions. We have performed the quantization of super BF theory in any dimension. The interesting question is thus how (and which) supergravity theories can be described as constraint super BF theories. The approach would then be as in popular models of four-dimensional quantum gravity (for reviews, see $[2,3]$ ). Namely, one tries to implement the constraints directly in the quantized theory, turning quantum super BF theory into quantum supergravity. A delicate issue in this context is to understand how to write supergravities as gauge theories of supersymmetically extended Lorentz groups when supersymmetry is an extension of the Poincaré group. (There is also the problem of giving a Poincaré invariant formulation for even dimensional theories.) It looks as if this direction might finally allow for a connection between spin foam quantum gravity and superstring theory. If feasible, this could provide the starting point for an approach to M-theory that is both manifestly background inependent and non-perturbative.

Many aspects of the present work need further investigation. For instance, one should check the asymptotics of the supersymmetric $6 j$-symbols and see if one can recover a Regge action for gravity+fermions. Extending this to the context of a cosmological constant, one should compute the $6 j$ symbol for the q-deformed $\mathrm{OSp}_{q}(1 \mid 2)$ and also compute its asymptotics in order to check the relation between $q$ and the cosmological constant $\Lambda$.

The super-Ponzano-Regge model as well as the higher dimensional quantum super BF theories provide invariants of manifolds that are potentially new. As already mentioned the relevant state sums are likely to be more convergent than the classical Ponzano-Regge model. However, there are but indications for now and this really needs to be investigated. The invariants might be related to the Casson invariant as is suggested by Witten's description of such a relation for similar supersymmetric theories [44]. However, at present this issue is not clear to us. 


\section{Acknowledgements}

E. L. would like to thank Yi Ling and Daniele Oriti, with whom he had started the project of studying three-dimensional supergravity, for many discussions on this subject. R. O. thanks John Barrett for discussions. He was supported by a Marie Curie Fellowship of the European Union.

\section{A Partition Functions for the Ponzano-Regge model}

\section{A.1 The original model: the graded partition function}

We define the Racah symbol as a recoupling coefficient (with $\Delta_{j}=2 j+1$ the dimension of the $j$-representation of $\mathfrak{s u}(2))$ :

$$
\begin{aligned}
& \left|\left(j_{1},\left(j_{2}, j_{3}\right) j_{23}\right) j\right\rangle= \\
& \sum_{j_{12}}\left(\Delta_{j_{12}} \Delta_{j_{23}}\right)^{1 / 2}(-1)^{j_{1}+j_{2}+j_{3}+j}\left\{\begin{array}{ccc}
j_{1} & j_{2} & j_{12} \\
j_{3} & j & j_{23}
\end{array}\right\}_{R}\left|\left(\left(j_{1}, j_{2}\right) j_{12}, j_{3}\right) j\right\rangle,
\end{aligned}
$$

which leads to the expression in terms of the Clebsh-Gordan coefficients

$$
\begin{aligned}
\left\{\begin{array}{ccc}
j_{1} & j_{2} & j_{12} \\
j_{3} & j & j_{23}
\end{array}\right\}_{R}=(-1)^{s i g n} \sum_{m_{1}, m_{2}, m_{3}} & {\left[\begin{array}{ccc}
j_{1} & j_{2} & j_{12} \\
m_{1} & m_{2} & m_{12}
\end{array}\right]\left[\begin{array}{ccc}
j_{12} & j_{3} & j \\
m_{12} & m_{3} & m
\end{array}\right] } \\
& {\left[\begin{array}{ccc}
j_{2} & j_{3} & j_{23} \\
m_{2} & m_{3} & m_{23}
\end{array}\right]^{*}\left[\begin{array}{ccc}
j_{1} & j_{23} & j \\
m_{1} & m_{23} & m
\end{array}\right]^{*} }
\end{aligned}
$$

with sign $=j_{1}+j_{2}+j_{3}+j$. Then using the (Biedenharn-Elliot) identity

$$
\begin{aligned}
& \left\{\begin{array}{ccc}
g & h & j \\
e & a & d
\end{array}\right\}_{R}\left\{\begin{array}{ccc}
g & h & j \\
e^{\prime} & a^{\prime} & d^{\prime}
\end{array}\right\}_{R}= \\
& \sum_{x} \Delta_{x}(-1)^{\operatorname{sign}}\left\{\begin{array}{ccc}
a & a^{\prime} & x \\
d^{\prime} & d & g
\end{array}\right\}_{R}\left\{\begin{array}{ccc}
d & d^{\prime} & x \\
e^{\prime} & e & h
\end{array}\right\}_{R}\left\{\begin{array}{ccc}
e & e^{\prime} & x \\
a^{\prime} & a & j
\end{array}\right\}_{R}
\end{aligned}
$$

with sign $=g+h+j+e+a+d+e^{\prime}+a^{\prime}+d^{\prime}+x$, and the corresponding $1 \leftrightarrow 4$ identity, one can easily show that the following model is topological:

$$
Z_{P R}=\sum_{\{j\}} \prod_{\text {edges }}(-1)^{2 j} \Delta_{j} \prod_{\text {triang }}(-1)^{a+b+c} \prod_{\text {tetra }}\{6 j\}_{R} .
$$




\section{A.2 The standard ungraded model}

One can use the Wigner 3j-symbols:

$$
\left(\begin{array}{ccc}
j_{1} & j_{2} & j_{3} \\
m_{1} & m_{2} & m_{3}
\end{array}\right)=\frac{(-1)^{j_{1}-j_{2}-m_{3}}}{\sqrt{\Delta_{j_{3}}}}\left[\begin{array}{ccc}
j_{1} & j_{2} & j_{3} \\
m_{1} & m_{2} & -m_{3}
\end{array}\right]
$$

to define the $6 \mathrm{j}$-symbol:

$$
\begin{aligned}
\left\{\begin{array}{lll}
j_{1} & j_{2} & j_{3} \\
j_{4} & j_{5} & j_{6}
\end{array}\right\}=\sum_{m_{i}}(-1)^{\text {sign }} & \left(\begin{array}{ccc}
j_{1} & j_{2} & j_{3} \\
m_{1} & m_{2} & m_{3}
\end{array}\right)\left(\begin{array}{ccc}
j_{5} & j_{6} & j_{1} \\
m_{5} & -m_{6} & m_{1}
\end{array}\right) \\
& \left(\begin{array}{ccc}
j_{6} & j_{4} & j_{2} \\
m_{6} & -m_{4} & m_{2}
\end{array}\right)\left(\begin{array}{ccc}
j_{4} & j_{5} & j_{3} \\
m_{4} & -m_{5} & m_{3}
\end{array}\right)
\end{aligned}
$$

with the sign $=j_{4}+j_{5}+j_{6}+m_{4}+m_{5}+m_{6}$ due to using the dual representation for $j_{4}, j_{5}, j_{6}$. The partition function for the Ponzano-Regge model is then simply:

$$
Z_{P R}=\sum_{\{j\}} \prod_{\text {edges }} \Delta_{j} \prod_{\text {tetra }}\{6 j\}
$$

If one writes the partition function for a closed 3-manifold made out of two identical tetrahedra glued together, one can check that

$$
\{6 j\}_{R}^{2}=\{6 j\}^{2},
$$

so the difference boils down to a difference in signs.

\section{References}

[1] JC Baez, An Introduction to Spin Foam Models of Quantum Gravity and BF Theory, Lect.Notes Phys. 543 (2000) 25-94, gr-qc/9905087

[2] D Oriti, Spacetime geometry from algebra: spin foam models for nonperturbative quantum gravity, Rept.Prog.Phys. 64 (2001) 1489-1544, grqc/0106091

[3] A Perez, Spin Foam Models for Quantum Gravity, Class.Quant.Grav. 20 (2003) R43, gr-qc/0301113

[4] R Oeckl, Generalized Lattice Gauge Theory, Spin Foams and State Sum Invariants, J.Geom.Phys. 46 (2003) 308-354, hep-th/0110259

[5] A Mikovic, Spin Foam Models of Matter Coupled to Gravity, Class.Quant.Grav. 19 (2002) 2335-2354, hep-th/0108099 
[6] L Crane, A New Approach to the Geometrization of Matter, grqc/0110060

[7] D Oriti, H Pfeiffer, A spin foam model for pure gauge theory coupled to quantum gravity, Phys.Rev. D66 (2002) 124010, gr-qc/0207041

[8] A Mikovic, Spin Foam Models of Yang-Mills Theory Coupled to Gravity, Class.Quant.Grav. 20 (2003) 239-246, gr-qc/0210051

[9] G Ponzano, T Regge, Semiclassical limit of Racah coefficients, Spectroscopic and Group Theoretical Methods in Physics (ed. F. Bloch et al.), North-Holland, Amsterdam, 1968

[10] H Ooguri, Partition Functions and Topology-Changing Amplitudes in the 3D Lattice Gravity of Ponzano and Regge, Nucl.Phys. B382 (1992) 276-304, hep-th/9112072

[11] A Achúcarro, PK Townsend, A Chern-Simons action for threedimensional Anti-De Sitter supergravity theories, Phys.Lett. B180 (1986) 89

A Achúcarro, PK Townsend, Extended Supergravities in $d=2+1$ as Chern-Simons theories, Phys.Lett. B229 (1989) 383

[12] JW Barrett, BW Westbury, Invariants of piecewise-linear 3-manifolds, Trans. Amer. Math. Soc. 348 (1996) 3997-4022

[13] L Crane, LH Kauffman, DN Yetter, State-Sum Invariants of 4Manifolds, J. Knot Theory Ram. 6 (1997) 177-234

[14] L Freidel, K Krasnov, Discrete Space-Time Volume for 3-Dimensional BF Theory and Quantum Gravity, hep-th/9804185, Class.Quant.Grav. 16 (1999) 351-362

[15] L Freidel, K Krasnov, R Puzio, BF Description of Higher-Dimensional Gravity Theories, Adv.Theor.Math.Phys. 3 (1999) 1289-1324, hepth/9901069

[16] JW Barrett, L Crane, Relativistic spin networks and quantum gravity, J.Math.Phys. 39 (1998) 3296-3302, gr-qc/9709028;

JW Barrett, L Crane, A Lorentzian Signature Model for Quantum General Relativity, Class.Quant.Grav. 17 (2000) 3101-3118, gr-qc/9904025

[17] Y Ling, L Smolin, Eleven dimensional supergravity as a constrained topological field theory, Nucl.Phys. B601 (2001) 191-208, hepth/0003285 
[18] R Oeckl, Renormalisation of discrete models without background, Nucl.Phys. B657 (2003) 107-138, gr-qc/0212047

[19] R Oeckl, The quantum geometry of spin and statistics, J.Geom.Phys. 39 (2001) 233-252, hep-th/0008072

[20] E Witten, (2+1)-Dimensional Gravity as an Exactly Soluble System, Nucl.Phys. B311 (1988) 46

[21] T Thiemann, QSD IV : 2+1 Euclidean Quantum Gravity as a model to test $3+1$ Lorentzian Quantum Gravity, Class.Quant.Grav. 15 (1998) 1249-1280, gr-qc/9705018

[22] L Freidel, ER Livine, C Rovelli, Spectra of Length and Area in 2+1 Lorentzian Loop Quantum Gravity, Class.Quant.Grav. 20 (2003) 14631478, gr-qc/0212077

[23] F Girelli, R Oeckl, A Perez, Spin foam diagrammatics and topological invariance, Class.Quant.Grav. 19 (2002) 1093-1108, gr-qc/0111022

[24] VG Turaev, OY Viro, State sum invariants of 3-manifolds and quantum 6j-symbols, Topology 31 (1992) 865-902

[25] H Ooguri, Topological lattice models in four dimensions, Mod.Phys.Lett. A7 (1992) 2799-2810

[26] R Oeckl, Braided Quantum Field Theory, Commun.Math.Phys. 217 (2001) 451-473, hep-th/9906225

[27] C Rovelli, Partial observables, Phys.Rev. D65 (2002) 124013, grqc/0110035

[28] R Oeckl, The quantum geometry of supersymmetry and the generalized group extension problem, J.Geom.Phys. 44 (2002) 299-330, hepth/0106122

[29] M Scheunert, W Nahm, V Rittenberg, Irreducible representations of the $\operatorname{osp}(2,1)$ and $\operatorname{spl}(2,1)$ graded Lie algebras, J.Math.Phys. 18 (1977) 155

[30] P Minnaert, S Toshev, Racah Sum Rule and Biedenharn-Elliott Identity for the Super-Rotation 6-j symbols, J.Math.Phys. 35 (1994) 5057-5064, hep-th/9402040

[31] L Freidel, D Louapre, Diffeomorphisms and spin foam models, Nucl.Phys. B662 (2003) 279-298, gr-qc/0212001

[32] JC Baez, Spin foam models, Class. Quantum Grav. 15 (1998), 18271858. 
[33] R Oeckl, Schrödinger's cat and the clock: Lessons for quantum gravity, Class. Quantum Grav. 20 (2003) 5371-5380, gr-qc/0306007

[34] R Oeckl, A "general boundary" formulation for quantum mechanics and quantum gravity, Phys. Lett. B 575 (2003) 318-324, hep-th/0306025

[35] D Williams, JF Cornwell, The Haar Integral for Lie Supergroups, J.Math.Phys. 25 (1984) 2922

[36] Y Ling, L Smolin, Supersymmetric Spin Networks and Quantum Supergravity, Phys.Rev. D61 (2000) 044008, hep-th/9904016

[37] Y Ling, Introduction to supersymmetric spin networks, J.Math.Phys. 43 (2002) 154-169, hep-th/0009020

[38] T Thiemann, Kinematical Hilbert Spaces for Fermionic and Higgs Quantum Field Theories, Class.Quant.Grav. 15 (1998) 1487-1512, grqc/9705021

[39] J Roberts, Skein theory and Turaev-Viro invariants, Topology 34 (1995) 771-787

[40] HC Lee, RB Zhang, Lickorish Ibvariant and Quantum OSp(1|2), q$\operatorname{alg} / 9611020$

[41] HC Lee, RB Zhang, Geometry and Representations of the Quantum SuperGroup $O S p_{q}(1 \mid 2)$, math.QA/9804111

[42] S Davids, Semiclassical Limits of Extended Racah Coefficients, grqc/9807061, J.Math.Phys. 41 (2000) 924-943; A State Sum Model for (2+1) Lorentzian Quantum Gravity, gr-qc/0110114

[43] L Freidel, A Ponzano-Regge model of Lorentzian 3-Dimensional gravity, gr-qc/0102098, Nucl.Phys.Proc.Suppl. 88 (2000) 237-240

[44] E Witten, Topology-changing amplitudes in $(2+1)$-dimensional gravity, Nucl. Phys. B 323 (1989) 113-140 